DIVISION OF THE HUMANITIES AND SOCIAL SCIENCES

CALIFORNIA INSTITUTE OF TECHNOLOGY

PASADENA, CALIFORNIA 91125

THE STRATEGY-PROOF PROVISION OF PUBLIC GOODS UNDER CONGESTION AND CROWDING PREFERENCES

Matthew O. Jackson

California Institute of Technology

Antonio Nicolò

University of Padova

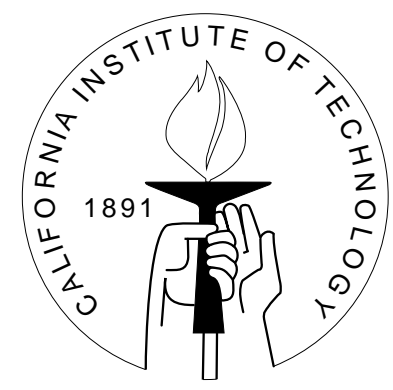

SOCIAL SCIENCE WORKING PAPER 1148

November 2002 


\title{
The Strategy-Proof Provision of Public Goods under Congestion and Crowding Preferences
}

\author{
Matthew O. Jackson Antonio Nicolò
}

\begin{abstract}
We examine the strategy-proof provision of excludable public goods when agents care not only about the level of provision of a public good, but also the number of consumers. We show that on such domains strategy-proof and efficient social choice functions satisfying an outsider independence condition must be rigid in that they must always assign a fixed number of consumers, regardless of individual desires to participate. The fixed number depends on the attitudes of agents regarding group size - being small when congestion effects dominate (individuals prefer to have fewer other consumers) and large when cost sharing effects dominate (agents prefer to have more consumers). A hierarchical rule selects which consumers participate and a variation of a generalized median rule to selects the level of the public good. Under heterogeneity in agents' views on the optimal number of consumers, strategy-proof, efficient, and outsider independent social choice functions are much more limited and in an important case must be dictatorial.
\end{abstract}

JEL classification numbers: D62, H23

Key words: public goods, congestion, club goods, strategy-proof 


\title{
The Strategy-Proof Provision of Public Goods under Congestion and Crowding Preferences*
}

\author{
Matthew O. Jackson
}

Antonio Nicolò

\section{Introduction}

In classic studies of the strategy-proof provision of public goods, public good consumption is assumed to occur without rivalry and without possibility of exclusion. That is, agents do not care about how many other agents consume the good, and all members of the society consume the public good. However, as Buchanan (1965) pointed out in his seminal work on club goods, in many cases of interest agents do care about who else consumes a public good and agents can be excluded from the use of the public good or else can freely decide not to consume the good. Examples of such goods are abundant, including for instance, public libraries, pools, and roads. In all of these cases agents prefer to have less crowded public good use, but at the same time they may also prefer to have costs of public facilities split among a larger pool of agents. Accounting for such preferences over who consumes a public good is an important aspect of evaluating the performance of mechanisms for the provision of public goods, especially for those goods whose use is not compulsory or can be regulated.

While the study of pure public goods as well as club goods is quite extensive (see Cornes and Sandler (1996)), the study of the strategy-proof provision is concentrated on the case of pure public goods. ${ }^{1}$ While the case of pure public goods is a useful starting point, the prevalence of some aspects of congestion and exclusion makes it imperative that the literature move beyond the case of pure public goods to understand the strategyproof provision of club goods as well. In this paper we study the provision of a club good; that is, an excludable public good when agents care about the number of other agents consuming the public good. We depart from the classic "single-peaked" pure public good

*Financial support under NSF grant SES-9986190 is gratefully acknowledged. We thank Salvador Barbera and David Cantala for helpful comments on an earlier draft.

${ }^{1}$ See Barbera (2001) for an up-to-date discussion of some of the literature on strategy-proofness. Some papers have considered exclusion as an incentive device (e.g., see Moulin (1994)), but none have examined congestion effects when exclusion is possible. Given that Groves schemes have a very abstract domain, one could use that framework to examine the issue (as we discuss below), but with some important differences from our analysis introduced by the transferable quasi-linear environment of the Groves analysis. 
model (e.g., Moulin (1980)) simply by allowing the group that consumes the public good to be a choice variable, and allowing individuals to have preferences over the size of this group.

In our model an allocation specifies a level of the public good (which might also be a location or some other attribute), as well as the group of agents who consume the public good. Agents have preferences over these allocation pairs. We focus on the case where agents have classic single-peaked preferences over the public good level, and consider several variations on how agents rank groups consuming the public good. One variation is the case of pure congestion, where agents prefer to have fewer people consuming the public good. This applies in situations where the distribution of any cost to producing the public good is either already fixed, or simply not an issue to begin with. Another variation is the other extreme where individuals would rather have more individuals consuming the public good. We refer to this as the case of pure cost-sharing, but it also includes some other applications as we discuss below. Both of these may be thought of as extreme cases of the more general setting where individuals have single-peaked preferences on group size. For instance, in the case of a toll road both effects are present: agents would prefer less congestion all else held equal, but also benefit from larger usage as that lowers the per capita cost or toll. In such situations agents may prefer an intermediate number of consumers of the public good. ${ }^{2}$

We characterize the strategy-proof and Pareto efficient social choice functions in these different cases; sometimes using auxiliary conditions such as a condition of 'outsider independence' which requires that the allocation not be dependent on the preferences of those not assigned to consume the good. ${ }^{3}$

One fundamental result that emerges is that strategy-proof and efficient rules must fix the size of the group assigned to consume the good and not allow this to vary with agents' preferences. In the case of pure congestion this amounts to assigning just one agent to the facility, and in the case of pure cost-sharing it amounts to assigning all agents to the public good; while in the general case where agents find groups of size $k$ to be optimal it amounts to assigning groups of size $k$ to the facility. This is true despite the fact that there are many efficient allocations in these settings which differ from such fixed size rules. We show how varying size is incompatible with strategyproofness. These results also imply that strategy-proofness and Pareto efficiency are generally incompatible with an individual stability notion that requires that individuals assigned to consume the public good weakly prefer consumption to abstinence, and those not assigned to consume weakly prefer not to consume. In fact, the strategy-proof and efficient rules can be incompatible with the weaker condition of individual rationality.

\footnotetext{
${ }^{2}$ The various aspects that affect agents' preferences over who consumes the public good may have to do with the actual consumption itself, or some arrangement about how payments are distributed among those consuming or not consuming the good. In our analysis we abstract away from the specifics generating the preferences and simply work directly with preferences over the size of the group, even though our labels may have some more specific connotations.

${ }^{3}$ This may be thought of as a weak version of a non-bossiness condition, with a normative grounding in self-determination.
} 
Another of the main results is that if there is heterogeneity in agents' preferences over the optimal sized group, then only dictatorial rules satisfy strategy-proofness and Pareto efficiency and outsider independence.

While some of the results are negative, in several important cases (e.g., cost sharing) there are still a variety of strategy-proof and Pareto efficient rules and the results provide partial or full characterizations of these rules depending on the preference domain.

\section{Relation to the Literature}

While there is an extensive literature on the strategy-proof provision of public goods ${ }^{4}$, ours is the first analysis (that we are aware of) that deals with the characterization of strategy-proof rules when the group assigned to consume the public good is something that agents care about and can be varied. The closest paper in spirit is by Bogomolnaia and Nicolò (1999) who consider a situation where agents have preferences that are singlepeaked over location and prefer less crowding for any fixed location. However, they study situations where two facilities must be located and each agent must be assigned exactly to one of these facilities. Although there is a dimension of preference over who else consumes at a particular location, the scope of those preferences, the applications, and the basic structure of the problem and results differ. Here, we focus on the problem of locating a single public good and where some group of agents must be chosen to participate, and remaining agents do not participate. This setting results in fairly dramatic differences in the structuring of strategy-proof and efficient rules compared to the two facility setting, and in fact stability is easily shown to be impossible in our setting while it is satisfied in the setting with two facilities. Also a goal of our analysis is to move beyond the case of congestion, and try to develop a general intuition for how preferences over number of co-users matter.

Our paper is also related to Cantala (2000) who analyzes the provision of a public good when agents can opt out of consumption. In his analysis, agents do not care about the group that consumes the good, but do care about its location and have reservation utilities. Since there are no congestion effects in Cantala's setting, the rules that Cantala identifies differ from ours and in particular allow the group that consumes the good to vary in size. The comparison of our results to Cantala's work is thus useful in deciphering the impact of congestion in the analysis. We shall see that congestion effects force us to fix the size of the group that consumes the good and force individual rationality to be violated.

The incompatibility of strategy-proofness with Pareto efficiency and individual rationality echoes a theme in the literature surrounding Groves' schemes (sometimes referred to as Clarke-Groves-Vickrey mechanisms, see Clarke (1971), Groves (1973) and Vickrey (1961)). There, strategy-proofness and an efficient level choice were incompatible with

\footnotetext{
${ }^{4}$ See Barberà (2000) for some discussion of the literature.
} 
balanced cost payments and individual rationality. ${ }^{5}$ It is important to emphasize that the nature of the incompatibilities, however, stems from quite different considerations and the settings and issues are also varied on several dimensions. Let us be more specific. First, in the Grovesian world, utility functions are quasi-linear and so Pareto efficiency ends up embodying a maximization of the sum of utility functions, and thus embodying cardinal preferences. This means that in that world, the allocation functions which are efficient are tightly defined and the incentive compatibility constraints that result are quite strong as differences in cardinal preferences must be uncovered. In our setting, utility is not assumed to be transferable, and as a result there are many more allocation functions which are Pareto efficient and so the incentive compatibility constraints are weaker in the sense that only ordinal information needs to be discovered. Second, in the Grovesian world a full domain of preferences is generally assumed, while here the preferences are assumed to be single-peaked. This restriction to single-peaked preferences provides an important reason why one might expect more positive results here, as it did in Moulin (1980). Third, the incompatibility in the Groves setting holds even without any congestive preferences or excludable nature of the public good, while here the fact that agents care about how many others are consuming the good is essential to the results. Fourth, transfers are admitted in the Grovesian analysis, while here transfers are not considered.

These differences on at least four dimensions, makes the current analysis and what we know from the Grovesian world hard to compare. The first two points describe ways in which the Grovesian analysis is more demanding, and the last two points describe ways in which our analysis is more demanding. Despite all of these differences, there is a basic intuitive level on which we can understand the similarity in conclusions. In the Groves setting, difficulties with individual rationality (or even balance) when combined with strategy-proofness and efficient decision making stem from the demands of uncovering fairly rich preference information - essentially cardinal information - and making choices that depend on that information. Here what we are seeing is also a strong demand in terms of uncovering preference information - but it is in terms of how agents tradeoff public goods for congestion. Having such detailed knowledge of preferences would be necessary to ensure voluntary participation, but turns out to be incompatible with incentives.

The richness of preferences coming from the added dimension of congestion effects is also the source of the contrast with the previous results on single-peaked domains. There Pareto efficient mechanisms could be run with very little knowledge of the details of agents preferences, beyond what their peaks are. In a sense, our results show how the positive results on single-peaked domains (e.g., Moulin (1980)) were dependent on the paucity of information about preferences that was needed to run the mechanisms (e.g., "peaks-only").

\footnotetext{
${ }^{5}$ See Green and Laffont $(1977,1979)$ and Laffont and Maskin (1980) for details in domains including public goods settings. Jackson (2001) provides an overview and some simple examples illustrating the incompatibilities.
} 
As a final remark before presenting the formal analysis, our model considers only the provision of a single good. That is, we do not allow agents who are excluded from consumption to form their own club and produce their own good. Our model fits cases where the public good is unique. For example, consider the case of a natural resource, like a natural park, when the agents have to decide the dimension of the area to be protected and the number of people (per year) allowed to visit the park. Individuals' preferences on the optimal number of visitors may depend from the tradeoff between the wish of preserving the natural environment, and the necessity of raising funds through the entrance fees in order to finance the public project. Also, this concentration on the provision of a single good allows us the most direct comparison to the literature on the strategy-proof provision of pure public goods, such as the important analysis of Moulin (1980). Nevertheless, there are cases outside of the scope of our model, such as the provision of local public goods under congestion, that fit well into the club goods setting and where the optimal number of goods to be provided is a natural issue. As such, our analysis should be viewed as a first step towards a more general analysis of the strategy-proof provision of club goods.

The paper proceeds as follows. We begin by providing basic definitions and outlining the preference domains we consider. Next, on these different domains we offer characterizations of rules satisfying strategy-proofness in combination with various other conditions such as Pareto efficiency, outsider independence, individual rationality and individual stability. All proofs are collected in the appendix.

\section{Notation and Definitions}

\section{A Society and Allocations}

A finite society of individuals, $N=\{1,2, \ldots, n\}$, chooses the location of a public facility and a set of individuals to use the public facility.

An allocation is a pair $(x, S)$ in $A=[0,1] \times 2^{N}$, where $x \in[0,1]$ specifies the location of the public facility and $S \subset N$ specifies the set of individuals assigned to the facility.

\section{Preferences}

Agent $i$ 's preferences over allocations are represented by a generalized single-peaked utility function $u_{i}: A \rightarrow \mathbb{R}$ that satisfies the following properties:

(1) there exists an $\widehat{x}_{i} \in[0,1]$ (agent $i$ 's peak) such that for all $x, y \in[0,1]$, if $\widehat{x}_{i} \geq y>x$ or $x>y \geq \widehat{x}_{i}$, then $u_{i}(y, S)>u_{i}(x, S)$ for all $S \subset N$ such that $i \in S$, 
(2) $u_{i}(x, S)=u_{i}(y, \emptyset)$ for all $(x, S)$ and $y \in[0,1]$ such that $i \notin S$.

(3) $u_{i}(x, S)=u_{i}\left(x, S^{\prime}\right)$ if $i \in S \cap S^{\prime}$ and $|S|=\left|S^{\prime}\right|$.

(4) $\exists(x, S) \in A$ such that $u_{i}(x, S)>u_{i}(x, \emptyset)$.

(5) $u_{i}$ is continuous. ${ }^{6}$

Without loss of generality, we normalize utility functions so that if $i \notin S$, then $u_{i}(x, S)=0$. Note that it could be the case that $u_{i}(x, S)<0$ for some $x \in[0,1]$ and for some $S \subset N$ with $i \in S$.

Item (1) states that preferences are single-peaked in the level of public good, and moreover that peak is independent of the group size. We talk about this independence in more detail below. Item (2) states that $i$ cares about differences in the location of the facility only if he or she is assigned to use the facility. Item (3) states that agents care only about the number of people who are assigned to the facility, but not the identity of the people in the group. Item (4) requires that there is some allocation that an agent prefers to not participating, which ensures that the public facility is indeed a public "good." Item (5) states that the preferences are continuous.

Given that the characterizations we obtain are very tight, the narrower the domain of preferences the stronger the results. Thus, the uniformity of the peak across groups (1) and the continuity (5) restrictions on preferences actually strengthen the results. Extensions to expand the domains are straightforward, as one needs only check that the stated rules in the results below remain strategy-proof and efficient when allowing for variance of the peak and/or discontinuous preferences. ${ }^{7}$

The set of utility functions satisfying the above conditions and normalization is denoted $U_{i}$.

Let $p\left(u_{i}\right) \in[0,1]$ denote agent $i$ 's peak under $u_{i}$.

Profiles, of utility functions are denoted $u=\left(u_{1}, \ldots, u_{n}\right)$. We denote by $\left(\hat{u}_{i}, u_{-i}\right)$ the vector $\left(u_{1}, \ldots, u_{i-1}, \hat{u}_{i}, u_{i+1}, \ldots, u_{n}\right)$. Let $U=U_{1} \times \cdots \times U_{n}$ be the set of profiles of generalized single-peaked utility functions.

In what follows, we consider several variations of sub-domains of preferences. Let us discuss some of them now.

\section{Congestion}

\footnotetext{
${ }^{6}$ Given that $2^{N}$ is finite, this amounts to requiring that $u_{i}$ is continuous in $x$ fixing any $S$.

${ }^{7}$ Of course, if one expands the domain too much, the results eventually become impossibility theorems, as should be expected.
} 
The first subdomain of $U_{i}$ is where agents experience congestion. An agent prefers to have fewer agents consume the public good. Let $U_{i}^{\text {cong }}$ denote the set of utility functions $u_{i} \in U_{i}$ satisfying the following condition:

(6) If $i \in S \cap S^{\prime}$ and $|S|<\left|S^{\prime}\right|$, then $u_{i}(x, S)>u_{i}\left(x, S^{\prime}\right)$ for any $x \in[0,1]$.

The corresponding subdomain of $U$ is denoted $U^{\text {cong }}$.

\section{Cost-sharing}

Another subdomain of $U_{i}$ of particular interest is one where agents prefer to have larger groups consume the public good. Let $U_{i}^{\text {shar }}$ be the set of utility functions $u_{i} \in U_{i}$ such that

(7) for all $S, S^{\prime} \in\left(2^{N}\right)$ such that $i \in\left(S \cap S^{\prime}\right)$, if $|S|>\left|S^{\prime}\right|$, then $u_{i}(x, S)>u_{i}\left(x, S^{\prime}\right)$ for all $x \in[0,1]$.

The corresponding subdomain of $U$ is denoted $U^{\text {shar }}$.

Let us discuss some applications covered under the cost-sharing subdomain.

One possibility is that a group is building and locating a facility which provides a public service or good. Here $x$ represents the location of the facility and $S$ the group of users. The cost of the public facility is to be shared equally among the users of the public facility. Here the only difference from the classic (excludable) public good problem is that individuals prefer to have more agents involved in order to lower their cost.

We remark that the preference domain $U$ requires that the peaks be independent of the size of the group consuming the public good. If instead, we consider a situation where the level of congestion of the public good depends not only on the number of consumers, but also on the size of the public good, then it could be that an individual's peak varies with the number of agents consuming the good. We note that the closure of that domain of preferences includes the domain of preferences considered here and that the characterizations presented below in fact extend to that case. ${ }^{8}$

Another application that is covered in this subdomain, but does not actually involve any "costs" to the public good, is the following. Consider a group of nations which have to reach an agreement on some common standard. Each country has different optimal choice as to what standard should be involved, and no state can be obliged to subscribe the agreement (for example on air pollution emissions). At the same time, the larger is

${ }^{8}$ Such a domain would allow individual peaks to vary as a function of group size. The proofs we provide do not require that peaks be independent of group size, and could be modified accordingly. As our characterizations are tight on the smaller domain, they provide for stronger results and so we stick with the smaller domain. 
the number of countries which reach the agreement, the greater is the welfare of each country. Note that under this interpretation, individual rationality becomes a natural condition to impose on the allocation.

This type of preference may also be generated also by private goods in presence of a network effect. Consider for example the case of computer operating systems or technological standards in communications. The utility of using a certain good depends not only on its intrinsic characteristics, but also on the number of consumers of this particular good. In this case our model fits the decision problem of companies which want to merge and use the same technology, or nations that have to agree on the use of the same technological standard in communications. In that case the public decision is the choice of standard or technology.

\section{"Size $k "$ Preferences}

The cases of congestion and cost-sharing can be thought of as opposite extremes of situations where individuals have some preference over the number of individuals who consume a public good. Intermediate cases, or cases where both effects are present are also of interest.

Generally, let $U_{i}^{k}$ denote the set of preferences of agent $i$ that stipulate groups of size $k \in\{1, \ldots, n\}$ as the optimal size. That is, let $U_{i}^{k}$ be the set of utility functions $u_{i} \in U_{i}$ such that

(8) for all $S, S^{\prime} \in\left(2^{N}\right)$ such that $i \in\left(S \cap S^{\prime}\right)$, if $k \geq|S|>\left|S^{\prime}\right|$ or $\left|S^{\prime}\right|>|S| \geq k$, then $u_{i}(x, S)>u_{i}\left(x, S^{\prime}\right)$ for all $x \in[0,1]$.

\section{Social Choice Functions and Properties}

We provide the following definitions on the domain $U$. Corresponding definitions for any subdomain are analogous.

A social choice function is a function $f: U \rightarrow[0,1] \times\left(2^{N}\right)$.

It will often be useful to split a social choice function into its two component functions. We write $f(u)=\left(f^{L}(u), f^{G}(u)\right)$, where $f^{L}: U \rightarrow[0,1]$ specifies the location or level of the public good and $f^{G}: U \rightarrow 2^{N}$ specifies the group consuming the public good.

\section{Strategy-Proofness}

A social choice function $f$ is strategy-proof if $u_{i}(f(u)) \geq u_{i}\left(f\left(\hat{u}_{i}, u_{-i}\right)\right)$ for all $\hat{u}_{i} \in U_{i}$, $u \in U$, and $i \in N$.

\section{Pareto Efficiency}


An allocation $(x, S)$ is Pareto efficient at $u$ if there does not exist another allocation $\left(y, S^{\prime}\right) \in A$ such that for $u_{i}\left(y, S^{\prime}\right) \geq u_{i}(x, S)$ for all $i$ with strict inequality for some $i$.

A social choice function is Pareto efficient if $f(u)$ is Pareto efficient for all $u \in U$.

\section{Individual Rationality}

An allocation $(x, S)$ is individually rational at $u$ if for all $i \in S, u_{i}(x, S) \geq 0$.

A social choice function is individually rational if $f(u)$ is individually rational at every $u \in U$.

Individual rationality requires that individuals who are assigned to consume the public good, should be willing to participate in the public good consumption. The expression here reflects our normalization of non-participation to a utility of 0 , and is equivalent to stating that individuals who are called to participate, weakly prefer to participate.

\section{Individual Stability}

An allocation $(x, S)$ is individually stable at $u$ if $u_{i}(x, S) \geq 0$ for all $i \in S$ and $0 \geq u_{j}(x,\{S \cup j\})$ for all $j \notin S$.

A social choice function is individually stable if $f(u)$ is individually stable for all $u \in U$.

Individual stability is a stronger condition than individual rationality. In addition to requiring that those who are participating weakly prefer participation to nonparticipation, it also requires that those excluded from participating do not wish to participate. As we shall see, this is not an easy condition to satisfy in conjunction with strategy-proofness and Pareto efficiency. It is however, a condition that can be satisfied on its own and in conjunction with Pareto efficiency.

Lemma 1 For any $x \in[0,1], k \in N$, and $u \in U^{k}$, there exists $S \in 2^{N}$ such that $(x, S)$ is individually stable at $u$. Moreover, if $k=n$ (cost sharing) then there is a maximal such group (i.e., a superset of any other individually stable group). ${ }^{9}$

The proof of the lemma is simple. For instance in the case of cost sharing it works as follows. We order agents in non-decreasing order based on the minimum size group needed before an agent would prefer to consume the public good. We then look for the highest ordered agent for whom the number of agents up to him in the ordering is at least as large as that agent's threshold number. That uniquely determines a largest

\footnotetext{
${ }^{9}$ This unique super-group property also holds for any $k<n$ at any $u$ such that no more than $k$ individuals have $u_{i}(x, S) \geq 0$ when $|S|=k$ and $i \in S$. This is shown in the proof in the appendix. The condition can fail at some other $u \in U^{k}$ for any $k<n$, as Example 2 below shows.
} 
individually stable group. The largest such group is then easily seen to be Pareto efficient subject to the choice of $x$. A careful choice of $x$ then results in an efficient and individually stable rule.

The following example shows that the uniqueness of the maximal sized individually stable group only holds on the domain of cost sharing.

Example 2 Non-unique maximal size stable groups when $k<n$.

Fix some $x$ and let each $i$ have $u_{i}(x, S)>0$ if $|S|=k$ and $i \in S$, and $u_{i}(x, S)<0$ otherwise. If $k>1$, then the set of individually stable groups is any group of size $k$ as well as the empty group. If $k=1$ then the set of individually stable groups are the singletons.

The next example shows that if we move to a domain where there is heterogeneous preferences over group size then there may not exist any individually stable group.

Example 3 Non-existence of stable groups under heterogeneous size preferences.

Fix $x$ and let $n=2$. It will be clear that this extends to $n>2$ and more general preferences. Let $u_{1}(x,\{1\})>0>u_{1}(x,\{1,2\})$ and $u_{2}(x,\{1,2\})>0>u_{2}(x,\{2\})$.

Here $\{1\}$ is not individually stable as then 2 would prefer to consume. $\{1,2\}$ is also not individually stable as then 1 would prefer not to consume. Finally, $\{2\}$ is not individually stable as 2 would prefer not to consume alone.

\section{Outsider Independence}

In many cases we work with a condition that requires that a social choice function should not be dependent on changes in preferences of individuals who are not consuming the public good. This can be viewed as a weak version of a non-bossiness condition, and is all that is needed in most of the characterizations and has an easy interpretation. ${ }^{10}$

A social choice function $f$ is outsider independent if for all $i \in N, u \in U$ and $u_{i}^{\prime} \in U_{i}$, if $i \notin S$ and $i \notin S^{\prime}$ where $(x, S)=f(u)$ and $\left(x^{\prime}, S^{\prime}\right)=f\left(u_{i}^{\prime}, u_{-i}\right)$, then $f(u)=f\left(u_{i}^{\prime}, u_{-i}\right)$.

Outsider independence requires that decisions regarding the public good be made by those involved in the consumption. The condition rules out some social choice functions,

\footnotetext{
${ }^{10}$ The stronger standard condition of non-bossiness is as follows. A social choice function $f$ is nonbossy if for all $i \in N, u \in U$ and $\bar{u}_{i} \in U_{i}, u_{i}(f(u))=u_{i}\left(f\left(\bar{u}_{i}, u_{-i}\right)\right)$ implies $u_{j}(f(u))=u_{j}\left(f\left(\bar{u}_{i}, u_{-i}\right)\right)$ for all $j \in N$. Outsider independence is the equivalent definition in our environment of a condition called non-bossiness of excluded individuals by Deb and Razzolini (1999).
} 
but we argue that the rules that fail to satisfy outsider independence are less attractive than the counterparts that are admitted. We provide some examples below.

While outsider independence is a reasonable condition, our use of it is largely driven by its usefulness in the proofs of the characterization results. It may in fact be unnecessary in some of the results, but we have not found a method around it.

\section{Characterization Theorems}

We begin by reminding the reader of the class of social choice functions that are strategyproof when agents do not care about the number of agents consuming the public good, and all agents are assigned to consume. ${ }^{11}$ This is the class of generalized median voting rules identified by Moulin (1980).

For each $C \subset N$ (including $C=\emptyset$ ) choose $a_{C} \in[0,1]$, such that $a_{C} \geq a_{C^{\prime}}$ when $C \subset C^{\prime}$. Let

$$
f^{L}(u)=\min _{C \subset N}\left(\max _{i \in C}\left[a_{C}, p\left(u_{i}\right)\right]\right) .
$$

This is the full class of social choice functions that are strategy-proof and have a connected range. ${ }^{12}$

\section{Sovereign Generalized Median Rules}

There is a special class of generalized median rules that play an important role in our characterizations. This is the class where $a_{C} \in\{0,1\}$ for all $C \subset N$, and where $a_{N}=0$ and $a_{\emptyset}=1$. We call this class the sovereign generalized median rules.

This class has two special properties:

(i) it has a full range of public good levels, and

(ii) it always picks the peak of some agent.

It is easy to verify that this subclass is in fact characterized by these properties. ${ }^{13}$

The sovereign generalized median rules include dictatorial rules (setting $a_{C}=0$ if the dictator $i$ is in $C$ and $a_{C}=1$ otherwise), median voting rules (setting $a_{C}=0$ if $C$ has

\footnotetext{
${ }^{11}$ That is equivalent to the class of rules where $f^{G}(u)$ is fixed to be $N$ for all $u$ and only $f^{L}$ is varied, as then preferences over group size are irrelevant.

${ }^{12}$ For a proof of this see Barberà and Jackson (1994). The class with arbitrary ranges is a variation on this definition and is also discussed in Barberà and Jackson (1994). For more on the role of the range and other interpretations of such generalized median rules see Barberà, Masso and Neme (1997).

${ }^{13}$ This class of rules also plays an important role in the characterizations of Cantala (2000) who calls them extreme minimax rules.
} 
at least half of the agents and $a_{C}=1$ otherwise), rules found according to other order statistics of peaks, as well as a variety of other rules including ones that give special consideration to some coalitions of agents.

An important subclass of generalized median rules that are not admitted in the sovereign generalized median rules are those including (interior) "phantom voters," as termed by Moulin (1980). We point out below why such phantom voting rules fail to satisfy strategy-proofness and efficiency on domains where agents have preferences over the number of consumers.

\subsection{The Cost-Sharing Domain}

We begin by characterizing the strategy-proof social choice functions on the cost sharing domain, since this is the most closely related domain to the classic domain where agents do not care about the number of agents consuming the public good.

On the cost-sharing domain all agents prefer to have more agents consuming the good rather than fewer and so the only difference between this domain and the classic domain is that it is possible that some agents might prefer not to participate. For instance, consider a situation where two thirds of the agents have a peak at 0 and the remaining agents have a peak at 1 , and where agents prefer not to participate if the location is more than 1/4 away from their peak, but prefer to participate at their peak no matter how many others participate. Here, one might think that a reasonable allocation would be to locate the public facility at 0 and then have the two thirds of the agents whose peaks are at 0 participate. Even though this is a Pareto efficient allocation, it cannot be in the range of a strategy-proof and efficient social choice function.

The following theorem shows that in fact any strategy-proof and efficient social choice function must always assign the complete group to consume the public good, even if some would prefer not to participate.

Theorem 4 A social choice function $f: U^{\text {shar }} \rightarrow A$ is strategy-proof, Pareto efficient, and outsider independent if and only if it always assigns the whole group to the facility and selects the location via a sovereign generalized median rule.

The proof of Theorem 4 appears in the appendix.

It is not clear that outsider independence is needed in the characterization established in Theorem 4. We have not found a proof without it nor an example showing that the theorem fails with only strategy-proofness and Pareto efficiency on the sharing domain.

As mentioned before, the sovereign generalized median rules do not include the rules based on phantom voters where some phantom is in the interior. To get an idea of why the phantom rules fail strategy-proofness and efficiency in this setting, consider 
$n=2$ and a rule which selects the median of $\left[p\left(u_{1}\right), p\left(u_{2}\right), \frac{1}{2}\right]$. This rule is strategy-proof and constrained Pareto efficient under the constraint that all agents be assigned to the facility. However, it is not Pareto efficient. For instance, If $p\left(u_{1}\right)=0$ and $p\left(u_{2}\right)=1$ and $u_{i}\left(\frac{1}{2},\{1,2\}\right)<0$, then both agents would be better off not participating! So, the only sovereign rules that are strategy-proof and Pareto efficient on $U^{\text {shar }}$ with $n=2$ are the rule that picks the max of the peaks, the rule which picks the min of the peaks, and the rules that are dictatorial. More generally, the theorem tells us that a strategy-proof and Pareto efficient rule must pick some peak at every preference profile.

Given that all agents must be assigned to consume in any strategy-proof and efficient social choice function on the cost sharing domain, it follows that there will be cases where some agents would prefer not to consume and hence individually rationality will be violated. This is easily seen by considering a preference profile where some agents have a peak at 0 and are only happy to participate if the public good level is near 0 and others have a peak at 1 and are only happy to participate if the public good level is near 1. Regardless of the level of public good chosen some agent will prefer not to participate. This reasoning leads to the following corollary.

Corollary 5 There does not exist a social choice function $f: U^{\text {shar }} \rightarrow$ A that is strategyproof, Pareto efficient, outsider independent, and individually rational; and thus there is no strategy-proof, Pareto efficient, outsider independent, and individually stable social choice function.

To get a better feeling for why there is no strategy-proof social choice function that varies the group of participants, consider the following natural rule and let us see why it is not strategy-proof even though it is Pareto efficient and individually stable.

\section{Example 6 A Voluntary Participation Rule}

Consider the following social choice function for the case of $n=2$. (Similar examples can be constructed for more agents.) With each $x \in[0,1]$ associate the largest individually stable group at the chosen location. Such a group exists and is unique by Lemma 1. Let agent 1 pick from these allocations. ${ }^{14,15}$

To see that such a rule is not strategy-proof, consider $u \in U^{\text {shar }}$ where agents have peaks $p\left(u_{1}\right)=0, p\left(u_{2}\right)=1$. Suppose that agent 1 prefers to participate with any sized group and has preferences such that $u_{1}(x, S)>u_{1}\left(x^{\prime}, S^{\prime}\right)$ whenever $1 \in S$ and $|S|>\left|S^{\prime}\right|$.

\footnotetext{
${ }^{14}$ Given the continuity of preferences, and the preference for larger sized groups, points of discontinuity in the largest stable group have larger groups and so this is well-defined. For example, it may be that $\{1,2\}$ is individually stable for all $x \in[a, b]$ and then $\{2\}$ is stable on $(b, c]$ and then $\emptyset$ is individually stable on $(c, 1]$. This follows from the upper-hemicontinuity of the correspondence of individually stable groups as $x$ is varied.

${ }^{15}$ Note that simply picking a peak according to some rule and then a largest individually stable group at that peak is not Pareto efficient. For instance suppose that the choice is $\left(p\left(u_{1}\right),\{1\}\right)$, while at some other $x$ both agents wish to participate. It could be that both are better off with the second choice.
} 
So, agent 1 cares more about group size than location of the public good. Let agent 2 have preferences so that $\bar{u}_{2}(x, S) \geq 0$ if and only if $S=N$ and $x \geq \frac{2}{3}$. Then, under truthful announcements by the agents the outcome is $\left(\frac{2}{3},\{1,2\}\right)$. If agent 2 lies and announces $\bar{u}_{2}$ such that $u_{2}(x, S) \geq 0$ if and only if $S=N$ and $x \geq \frac{3}{4}$, then the outcome will be $\left(\frac{3}{4},\{1,2\}\right)$. Agent 2 is better off announcing $\bar{u}_{2}$ when his true preference is $u_{2}$, and so the rule is not strategy-proof.

An implication of Theorem 4 and Corollary 5 is that in order to have a strategy-proof and Pareto efficient provision of the public good, there must be some enforcement of the mechanism as individuals will not always wish to participate when they are called on to do so. This is a property that carries over to any domain where agents have some preference over group size, as we shall see below.

Before proceeding, we note that, excepting for the role of outsider independence, Theorem 4 is tight. If one drops strategy-proofness, then rules such as the voluntary participation rule in Example 6 satisfy efficiency. If one drops Pareto efficiency, then other strategy-proof rules appear. For instance, one can fix the group to be any $S$ and then run any generalized median rule to select the location (and the generalized median rule could pay attention to individuals who are not in $S$ ).

To get a better feeling for the strategy-proof but inefficient rules, we conclude this section with the following result which characterizes what happens when we replace Pareto efficiency with individual stability.

Theorem 7 If $f: U^{\text {shar }} \rightarrow A$ is strategy-proof, outsider independent and individually stable, then there exists $a \in[0,1]$ such that for each $u \in U^{\text {shar }}, f(u)=(a, S)$ where $S$ is individually stable at $u$ relative to a.

Theorem 7 provides a dual to Theorem 4 . Theorem 4 shows that strategy-proofness and efficiency require one to fix the group of users (to be the entire set of agents) and to perform a suitable general median voter rule to locate the facility. Theorem 7 shows that strategy-proofness and individual stability (plus outsider independence) require us to fix the location of the facility and then choose an individually stable set of agents to be the set of users.

The following examples provide some insights on Theorem 7 . Example 8 clarifies the role of outsider independence in Theorem 7 .

Example 8 Role of Outsider Independence

Let $S_{x}(u)$ be the maximal sized individually stable group at $u \in U^{\text {shar }}$ relative to $x$. Define $f$ as follows. Let $f(u)=\left(0, S_{0}(u)\right)$ if $u_{2} \in U_{2}^{\text {shar }}$ is such that $p\left(u_{2}\right)=\frac{1}{2}$ and $u_{2}(x, S) \geq 0$ only if $x \in\left[\frac{1}{3}, \frac{2}{3}\right] ;^{16}$ and let $f(u)=\left(1, S_{1}(u)\right)$, otherwise. This rule violates

\footnotetext{
${ }^{16}$ Note that agent $2 \notin S_{0}(u)$.
} 
outsider independence, but it is strategy-proof and individually stable. According to this rule the facility is not always located at the same place.

The next example shows why the converse to Theorem 7 requires one to be careful in selecting the group.

Example 9 Selection of the stable group

Let $N=\{1,2\}$. Consider the following rule. For all $u \in U^{\text {shar }}$ let $f(u)=\left(\frac{1}{2}, S_{\frac{1}{2}}^{\min }\right)$ where $S_{\frac{1}{2}}^{\min }$ is a stable group with the minimum cardinality at $\frac{1}{2}$. This rule is outsider independent and individually stable, but not strategy-proof. To see this, consider a profile $u \in U^{\text {shar }}$ such that $u_{i}\left(\frac{1}{2},\{i\}\right)<0$ and $u_{i}\left(\frac{1}{2},\{1,2\}\right)>0$ for both $i=1,2$. It follows that $f(u)=\left(\frac{1}{2}, \emptyset\right)$. Let $\hat{u}_{1} \in U_{1}^{\text {shar }}$ be such that $\hat{u}_{1}\left(\frac{1}{2},\{1\}\right)>0$. Individual stability implies that $f\left(\hat{u}_{1}, u_{2}\right)=\left(\frac{1}{2},\{1,2\}\right)$, but then strategy-proofness is violated at $u$ by agent 1 via $\hat{u}_{1}$.

The previous example suggests that failing to pick the largest individually stable group may result in a violation of strategy-proofness. It is easily seen that always selecting the largest individually stable group at a fixed location will result in a strategy-proof rule. However, there are also some

other strategy-proof (outsider independent and individually stable) rules, as the final example of this section shows.

Example 10 Selection of the stable group II

Let $N=\{1,2,3\}$. Consider the following rule. For all $u \in U^{\text {shar }}$ let $f^{L}(u)=a$ and $f^{G}(u)$ is the largest individually stable group for which all agents have strictly positive utility in being assigned to the facility. Consider, for example, utility profile $u \in U^{\text {shar }}$ such that $u_{3}(a, N)=0$ and $u_{3}(a, S)<0$ for all $S \neq N$, while $u_{i}(a,\{i\})>0$ for both $i=1,2$. It follows that $f(u)=(a,\{1,2\})$, while the largest stable group at $a$ is $N$.

This rule is outsider independent, individually stable, and strategy-proof, even though it does not always select the largest stable group at the given location. ${ }^{17}$

\subsection{The Pure Congestion Domain}

We now turn to the other extreme domain of pure congestion, where agents all prefer to have smaller groups consume the public good.

\footnotetext{
${ }^{17}$ It is easy to show, however, that the freedom to vary the selection of the stable group is limited. In cases where all agents in the largest stable group at some given location get strictly positive utility from being in the largest stable group at that location, then the rule must pick the largest stable group. This is true regardless of how the location is chosen.
} 
The basic intuition that there will be problems in varying the group size in a strategyproof rule carries over to this case. However, in the case of congestion this suggests that the group size should be just 1, and hence we end up with only dictatorial rules. This is stated in the following theorem.

Theorem 11 A social choice function $f: U^{\text {cong }} \rightarrow A$ is strategy-proof, Pareto efficient, and outsider independent if and only if it is dictatorial. ${ }^{18}$

Theorem 11 does require outsider independence. If outsider independence is dropped, then some non-dictatorial rules emerge. For instance, in a three agent economy one can find a strategy-proof rule where either agent 1 dictates or agent 2 dictates, depending in some arbitrary way on the announcement of preferences by agent 3 . While we have not been able to find a proof, we conjecture that the only new rules admitted by dropping outsider independence amount to some variation on picking a dictator in a way that depends on outsiders' preferences.

The characterization is tight. If one drops Pareto efficiency, then fixing any group $S$ and running a generalized median rule on that group to choose the location results in a strategy-proof and outsider independent social choice function. If one drops strategyproofness then, for example, one admits rules of the following form. If both agents 1 and 2 have $u_{i}\left(p\left(u_{1}\right),\{1,2\}\right)>0$ then let the outcome be $\left(p\left(u_{1}\right),\{1,2\}\right)$, and otherwise let the outcome be $\left(p\left(u_{n}\right),\{n\}\right)$. As the set of efficient allocations is quite large, there are many Pareto efficient and outsider independent rules that are not strategy-proof.

\subsection{The Domain of a Common Optimal Group Size $k$}

We now turn to the more general case where both congestion and cost sharing effects may be present. As in the above extreme cases, strategy-proof, efficient and outsider independent rules cannot vary the group size. The full characterization of all such rules is complex and tedious, so we offer a partial characterization that captures most of the properties of the rules.

Theorem 12 Fix $k \in\{1, \ldots, n\}$. If a social choice function $f: U^{k} \rightarrow A$ is strategyproof, Pareto efficient, and outsider independent, then it always assigns a group of size $k$ to use the facility, the level of public good selected depends only on the peaks of the agents over levels, and at least one agent whose peak coincides with the level of the public good is included in the group of assigned consumers.

The theorem stops short of providing a complete characterization as it leaves partly open how the rules select among the peaks and how agents are assigned to the facility. A bit more can be seen fairly easily, as the fact that the peak selection must always

\footnotetext{
${ }^{18}$ There exists $i$ such that $f(u)=\left(p_{i}\left(u_{i}\right),\{i\}\right)$ for all $u \in U^{\text {cong }}$.
} 
be a peak of an agent assigned to the facility together with the properties of strategyproofness, efficiency, and outsider independence implies that the level is chosen according to a sovereign generalized median rule which depends only on the peaks of the agents who always may be assigned to consume if they so desire (and some such agents exist). However, a full characterization involves a system by which assignment of the group to use the facility can follow fairly complicated priority rules. To get a feeling for the complexity of the rules that can satisfy the above conditions, consider the following example.

\section{Example 13 Group Selection}

The level of the public good is the minimum of agent 1 and agent 2's peaks. The $n>k>1$ agents assigned to consume are chosen as follows. If agent 1's peak is the minimum, then select the first $k$ agents who have positive utility for being assigned to the facility (in a group of size $k$ ) by the following priority rule: $1,2,3, \ldots, n$. If there are less than $k$ agents with positive utility, select the remaining agents to be forced in the group by the following priority rule: $n, n-1, \ldots, 2$. If agent 2 's peak is the lowest then select the first $k$ agents who have positive utility in being assigned to the group by the following priority rule $2,1, n, n-1, \ldots, 3$. If there are less than $k$ agents select the agents to be forced in the group by the same priority rule.

Finally note that individual stability is a difficult condition to satisfy in conjunction with strategy-proofness. A direct corollary to Theorem 12 is that there does not exist any social choice function $f: U^{\mathrm{k}} \rightarrow A$ that is strategy-proof, Pareto efficient, outsider independent, and individually stable. ${ }^{19}$ The following stronger result is, in fact, true.

Theorem 14 If $k<n$, then there does not exist any social choice function $f: U^{\mathrm{k}} \rightarrow A$ that is strategy-proof and individually stable.

Note that Theorem 14 uses very little of the structure of the preference domain. It holds even if all agents have identical known peaks and we fix the location to be that peak. It results entirely from the selection of the group and agents preferences over group sizes versus non-participation. ${ }^{20}$

\subsection{Heterogeneous Most Preferred Group Sizes}

Theorem 12 only applies to situations where all agents have exactly the same optimal group size in mind. In many applications, it may be that agents disagree about the optimal group size, and their preferences over the optimal group size may not always be constant.

\footnotetext{
${ }^{19} \mathrm{As}$ with $k=n$, if $k>1$ one can show that in fact individual rationality must be violated.

${ }^{20} \mathrm{~A}$ similar result is noted in Bogomolnaia and Jackson (2002) in a pure coalition formation setting.
} 
What we show in the theorem below is that even a small expansion of the preference domain to allow for some variance in optimal group size leads to a very negative conclusion. The small expansion in the domain is simply to allowing an agent's favorite group size to take on more than one value.

Let $U^{k, k^{\prime}}=\times_{i}\left(U_{i}^{k} \cup U_{i}^{k^{\prime}}\right)$. This is a domain where agents' favorite sized groups may take on more than one value.

Theorem 15 Consider any group sizes $k$ and $k^{\prime}$ with $k \neq k^{\prime}$. If a social choice function $f: U^{k, k^{\prime}} \rightarrow[0,1] \times 2^{N}$ is strategy-proof, Pareto efficient, and outsider independent, then it is dictatorial. ${ }^{21}$

Theorem 15 does not provide a full characterization as we have not specified exactly how the group is selected and only certain methods of selecting the group are compatible with strategy-proofness. But in this case, a strategy-proof rule must identify a single agent $i$, locate the public good at that agent's peak, choose a group that includes $i$ and is of $i$ 's optimal size. The only thing that we have left unspecified in the characterization is the exact selection of the group, which again can follow a hierarchical priority system (as in Example 13). ${ }^{22}$

\section{Discussion}

When the number of consumers of a public good is an important aspect of agents' preferences, strategy-proofness, efficiency and individual stability are not compatible. In order for strategy-proofness, efficiency, and outsider independence to be satisfied, some agents must be either forced to consume the public good, or excluded from its usage. Thus, one of the implications of the results here is that in order to implement a strategy-proof and efficient social choice rule we do need a "planner" who has some coercive power over the set of possible consumers of the public good. It follows that for the provision of public goods when the size of the group of users matters, there must exist a real enforcing authority and not

just a virtual organization among its members.

Another thing to note about the results is that anonymity is naturally precluded, since even when all agents have the same preferences some group of size $k$ must be selected. This is partly due to our approach which only considers determinate groups of consumers as outcomes. It may be of interest to study partial exclusion and/or randomization in settings where some congestion is present.

\footnotetext{
${ }^{21}$ A social choice function is dictatorial if there exists $i \in N$ such that the outcome is always $i$ 's most preferred level and group size, with $i$ in the group.

${ }^{22}$ To get a feeling for how such priority systems can work in the face of strategy-proofness, see Barberà, Jackson and Neme (1997) who outline priority systems in allotment rules or Papai (2000) who outlines hierarchies in an allocation problem.
} 
Theorem 15 is somewhat pessimistic as it shows that allowing for flexibility in size will be incompatible with strategy-proofness unless one cares for dictatorial rules. Thus, efficiency and strategy-proofness are somewhat at odds here. However, if one allows for approximate efficiency, and for instance considers large economies where the distribution over preferences for group size is known, then fixing a group size may not be far from efficient, and would allow a variety of non-dictatorial, strategy-proof, and approximately efficient rules to be available.

We have focused on the provision of a single good. Studying the strategy-proof provision of more than one public good and possibly with side payments, but with preferences on group size, is an important area for future study. This is especially true, given that our results showing that the size of groups must be fixed. In cases where the preferred size $k$ is smaller than $n$, it is natural to think of producing several local public goods. This would involve bringing together approaches such as that here with those as in Bogomolnaia and Nicolò (1999) and Le Breton and Weber (2001), among others in the more general Tiebout and club goods literatures.

As one final note, the characterizations we have obtained here hold under even stricter domains of preferences. For instance, the proofs in the appendix all hold if we add a condition: $u_{i}(x, S)>u_{i}(y, S)$ if and only if $u_{i}\left(x, S^{\prime}\right)>u_{i}\left(y, S^{\prime}\right)$ whenever $i \in S \cap S^{\prime}$. This makes the results stronger as it implies that the characterizations are tight even with a separability in utility between $x$ and $S$. 


\section{References}

Barberà, S. (2001), "An Introduction to Strategy-Proof Social Choice Functions," Social Choice and Welfare, vol 18, pp 6??-654.

Barberà, S. and C. Bevia (2001) "Self- Selection Consistent Functions," forthcoming in Journal of Economic Theory.

Barberà, S. and M.O. Jackson (1994), "A Characterization of Strategy-proof Social Choice Functions for Economies with Pure Public Goods," Social Choice and Welfare, Vol. 11, pp. 241-252.

Barberà, S., M.O. Jackson and A. Neme (1997), "Strategy-Proof Allotment Rules," Games and Economic Behavior, Vol. 18, pp. 1-21.

Barberà, S., J. Masso, and A. Neme (1997), "Voting Under Constraints," Journal of Economic Theory, Vol. 76, pp. 298-321.

Berga, D. (1997), "Maximal Domains and Strategy-Proofness in Public Good Economies," thesis: Universitat Autonoma de Barcelona.

Bogomolnaia, A. and M.O. Jackson (2002), "The Stability of Hedonic Coalition Structures," Games and Economic Behavior, Vol. 38, No. 2, pp 201-230..

Bogomolnaia, A. and A. Nicolo (1999), "Stable assignment of public facilities under congestion" mimeo: Universitat Autonoma de Barcelona.

Buchanan, J.M. (1965) “An Economic Theory of Clubs," Economica Vol. 32, pp. 1-14.

Cantala, D. (2000) "Choosing a Level of Public Good when Agents have an Outside Option," mimeo: ITAM.

Clarke, E.H.(1971) "Multi-part Pricing of Public Goods," Public Choice,11,17-33.

Cornes, R. and T. Sandler(1996) The Theory of Externalities, Public Goods, and Club Goods, Cambridge University Press: Cambridge, U.K.

Deb, R. and L. Razzolini(1999), "Auction-Like Mechanisms for Pricing Excludable Public Goods," Journal of Economic Theory, Vol. 88, pp. 340-368. 
Green, J. and J.J. Laffont(1977) "Characterization of Satisfactory Mechanisms for the Revelation of Preferences for Public Goods," Econometrica, 45727-738.

Green, J. and J.J. Laffont(1979)Incentives in Public Decision MakingNorth Holland: Amsterdam.

Groves, T.(1973) "Incentives in Teams" Econometrica,41,617-663.

Jackson, M.O. (2001), "Mechanism Theory," Encyclopedia of Life Support Systems.

Laffont, J.J. and E. Maskin(1980) "A Differential Approach to Dominant Strategy Mechanism Design," Econometrica,48,1507-1520.

Le Breton, M. and S. Weber(2001), "The Art of Making Everybody Happy: How to Prevent a Secession," mimeo: Université d'Aix-Marseille and SMU.

Moulin, H. (1980), "On Strategy-Proofness and Single-Peakedness," Public Choice, Vol. 35, pp. 437-455.

Moulin, H.(1994) "Serial Cost Sharing of Excludable Public Goods" Review of Economic Studies,Vol. 61,pp. 305-325.

Papai, S. (2000), "Strategy-proof Assignment through Hierarchical Exchange," Econometrica, Vol. 68, pp. 1403-1434. 
Vickrey, W.(1961) "Counterspeculation, Auctions, and Competitive Sealed-Tenders," Journal of Finance, 16,pp 8-37.

\section{Appendix}

For any $i$ and $k$, let $U_{i}^{k+}$ denote the subdomain of $U_{i}^{k}$ such that $u_{i}(x, S)>0$ for all $(x, S)$ such that $i \in S$.

Proof of Lemma 1: Fix any location choice $x \in[0,1]$ and some $k \in N$ and $u \in U^{k}$. Let us first show that there always exists a choice of an $S$ for which $(x, S)$ is individually stable at $u$.

Case 1: No more than $k$ individuals have $u_{i}(x, S) \geq 0$ when $|S|=k$ and $i \in S$.

Let $m_{i}$ be such that $u_{i}(x, S) \geq 0$ when $|S|=m_{i}$ and $i \in S$, but $u_{i}(x, S)<0$ when $|S|<m_{i}$ and $i \in S$. (Set $m_{i}=n+1$ if $u_{i}(x, S)<0$ for all $S$ with $i \in S$ and $m_{i}=1$ if $u_{i}(x, S) \geq 0$ for all $S$ such that $i \in S$.) So, $m_{i}$ is the minimal sized group that $i$ is willing to be a member of at $x$. Note $m_{i}$ is uniquely determined under the preference assumptions. Order the agents in terms of $m_{i}$. In particular, without loss of generality assume that $m_{n} \geq m_{n-1} \geq \cdots \geq m_{1}$. Find the largest $i$ such that $i \geq m_{i}$. If there is no such $i$, then clearly the empty group is individually stable. Otherwise, it follows directly that $\{1, \ldots, i\}$ is individually stable.

Case 2: More than $k$ individuals have $u_{i}(x, S) \geq 0$ when $|S|=k$ and $i \in S$.

Let $M_{i}$ be such that $u_{i}(x, S) \geq 0$ when $|S|=M_{i}$ and $i \in S$, but $u_{i}(x, S)<0$ when $|S|>M_{i}$ and $i \in S$. (Set $M_{i}=0$ if $u_{i}(x, S)<0$ for all $S$ with $i \in S$.) So, $M_{i}$ is the maximal sized group that $i$ is willing to be a member of at $x$. Again, $M_{i}$ is uniquely determined under the preference assumptions. Without loss of generality assume that agents are labeled so that $M_{1} \geq M_{2} \geq \cdots \geq M_{n}$. If $n \leq M_{n}$, then the group $N$ is individually stable. Otherwise, find $i$ such that $i \leq M_{i}$ but $i+1>M_{i+1}$ and then $\{1, \ldots, i\}$ is clearly individually stable. Note that $i$ is uniquely determined under our ordering of agents, and that $i \geq k$ by the definition of this case.

To complete the proof let us show that when $k=n$ then there is an individually stable group that includes all other individually stable groups. First note that when $k=n$ we must always fall in case 1 . Suppose that the empty group is selected under the algorithm in case 1 . Then it is clear from the definition of the $m_{i}$ 's that no other group is individually stable. Next, consider the case where some $\{1, \ldots, i\}$ is the group selected by the algorithm in case 1 . Suppose that some other $S$ is individually stable where $j \in S$ for some $j>i$. Let $j$ be the largest indexed individual in $S$. It must be that $|S| \geq m_{j}$ by individual stability and the definition of $m_{j}$. By our ordering $m_{j} \geq m_{h}$ for 
any $h<j$. Thus, by the preferences it must be that $h<j$ strictly prefers to be in $S$ if $h \notin S$, and so by individual stability $h \in S$ for all $h<j$. Thus, $\{1, \ldots, j\}=S$. However, then $j=|S| \geq m_{j}$ which contradicts that fact that $i<j$ was the largest indexed agent for whom $i \geq m_{i}$.

The following Lemmas are useful in the remaining proofs.

For any $u_{-i}$ and any $k$

$$
O_{i}^{k}\left(u_{-i}\right)=\left\{(x, S) \mid(x, S)=f\left(\bar{u}_{i}, u_{-i}\right) \text { for some } \bar{u}_{i} \in U_{i}^{k}\right\} .
$$

So, $O_{i}^{k}\left(u_{-i}\right)$ is the option set of $i$; that is, the set of outcomes that $i$ can generate fixing the profile of preferences of the other agents. Note that strategy-proofness implies that for each $u \in U^{k}$ and for all $i \in N$,

$$
f(u) \in \operatorname{argmax}_{(x, S) \in O_{i}^{k}\left(u_{-i}\right)} u_{i}(x, S) .
$$

For any $x \in[0,1]$, let $I(x)$ denote a. When we refer to a specific agent $i \in N$, we write $I_{i}(x)$. Finally let $S_{k}$ denote any group of agents with cardinality $k$.

Lemma 16 Let $f: U^{k} \rightarrow[0,1] \times 2^{N}$ be strategy-proof, and consider any $u \in U^{k}$ and let $(a, Y)=f(u)$. For each $i \in Y$ there exists a closed set with non-empty interior containing a, I $\subset[0,1]$, such that for any closed neighborhood of a with non-empty interior $I_{i}(a) \subset I$, then there exists $u_{i}^{\prime} \in U_{i}^{k}$ such that

- $p\left(u_{i}^{\prime}\right)=a$,

- $u_{i}^{\prime}(a, Y)>0$ and $u_{i}^{\prime}(x, S)<0$ for all $x \notin I(a)$ and for all $S$ such that $i \in S$,

- $f\left(u_{i}^{\prime}, u_{-i}\right)=(a, S)$ with $|S|=|Y|$ and $i \in S$.

Proof of Lemma 16: If $|Y|=k$, then the Lemma follows directly. So consider the case where $k^{\prime}=|Y|>k\left(k^{\prime}=|Y|<k\right)$. By strategy-proofness and the conditions on preferences, it must be that $(a, S) \notin O_{i}^{k}\left(u_{-i}\right)$ for any $S$ such that $i \in S$ and $k^{\prime}>|S| \geq k$ $\left(k^{\prime}<|S| \leq k\right)$. In fact, by the continuity of preferences and strategy-proofness it follows that there exists some $\gamma>0$ such that $(b, S) \notin O_{i}^{k}\left(u_{-i}\right)$ for any $S$ such that $i \in S$ and $k^{\prime}>|S| \geq k\left(k^{\prime}<|S| \leq k\right)$, and any $b$ such that $|b-a|<\gamma$.

Select $u_{i}^{\prime} \in U_{i}^{k}$ such that $p\left(u_{i}^{\prime}\right)=a$ and $u_{i}^{\prime}(x, S) \geq 0$ only if $x \in I_{i}(a)$ and $i \in S$, with $u_{i}(a, S)>0$ whenever $i \in S$, and the length of $I_{i}(a)$ is less than $\frac{\gamma}{2}$.

Since $(b, S) \notin O_{i}^{k}\left(u_{-i}\right)$ for any $S$ such that $i \in S$ and $k^{\prime}>|S| \geq k\left(k^{\prime}<|S| \leq k\right)$ and any $b$ such that $|b-a|<\gamma$, it follows that the most preferred alternative in $O_{i}^{k}\left(u_{-i}\right)$ under $u_{i}^{\prime}$ is $(a, Y)$ or any $(a, S)$ with $|S|=|Y|$. The lemma follows directly. 
Lemma 17 Consider a strategy-proof and outsider independent $f$ defined on $U^{k}$ for some $k$, and consider $u \in U^{k}$, letting $f(u)=(a, Y)$. For all $i \notin Y$, there exists $u_{i}^{\prime} \in U_{i}^{k}$ with $p\left(u_{i}^{\prime}\right) \neq a$ such that $f\left(u_{-Y}^{\prime}, u_{Y}\right)=(a, Y)$ and $p\left(u_{j}^{\prime}\right) \neq p\left(u_{h}^{\prime}\right)$ for each $j \notin Y$ and $h \neq j$.

Proof of Lemma 17: Let $f(u)=(a, Y)$. Consider any $j \notin Y$. Suppose that either $p\left(u_{j}\right)=a$ or $p\left(u_{j}\right)=p\left(u_{h}\right)$ for some other agent $h \in N$. By continuity of the utility functions, for each $j \notin Y$ there exists a closed interval with non-empty interior ${ }^{23} I_{j}\left(p\left(u_{j}\right)\right)$ such that $u_{j}(x, S) \geq 0$ for all $x \in I_{j}\left(p\left(u_{j}\right)\right)$ and $S$ such that $j \in S$ and $|S|=k$. Let $u_{j}^{\prime} \in U_{j}^{k}$ be such that $p\left(u_{j}^{\prime}\right) \in I_{j}\left(p\left(u_{j}\right)\right), p\left(u_{j}^{\prime}\right) \neq p\left(u_{h}\right)$ for any $h \in N$, and $u_{j}^{\prime}(x, S) \geq 0$ only if $|S|=k$ and $x \in I_{j}^{\prime}\left(p\left(u_{j}\right)\right) \subset I_{j}\left(p\left(u_{j}\right)\right)$. Then by strategy-proofness and outsider independence $f\left(u_{j}^{\prime}, u_{-j}\right)=(a, Y)$. Repeat the argument for all $j \notin Y$, choosing at $u_{j}^{\prime}$ a different peak for each agent $j$.

Proof of Theorem 4: Theorem 12, which is proven below, implies that the whole group must always be assigned to consume the good. The problem then boils down to a characterization of the efficient and strategy-proof rules where size preferences do not matter and a peak of some agent is always picked. Following Moulin (1980) (and see Barbera and Jackson (1994) for details), this is easily seen to be the class of sovereign generalized median rules.

\section{Proof of Theorem 7:}

We first define the following utility function. For any $c \in[0,1]$ and $i \in N$ let $\tilde{u}_{i}^{c}$ be such that $p\left(\tilde{u}_{i}^{c}\right)=c$ and

1) $|S|>\left|S^{\prime}\right|$ with $i \in S \cap S^{\prime} \Rightarrow \tilde{u}_{i}^{c}(x, S)>\tilde{u}_{i}^{c}\left(y, S^{\prime}\right)$ for all $x, y \in[0,1]$.

2) $\tilde{u}_{i}^{c}(x, S)>0$ for all $x \in[0,1]$ and for all $S \in 2^{N}$ with $i \in S$.

The theorem follows from the two steps below.

Step 1: For all $u \in U^{\text {shar }}, f(u)=(c, Y)$ implies $f\left(\tilde{u}^{c}\right)=(c, N)$.

If $Y=N$ this follows easily, so consider the case where $Y \neq N$. Consider any $i \in Y$. By Lemma 16 we can assume that $f(u)=(c, Y)$ with $p\left(u_{i}\right)=c$, and $u$ is such that for each $i \in Y: u_{i}(x, S) \geq 0$ and $i \in S$ implies that $x \in I_{i}(c)=I(c)$. By Lemma 17 assume that for each $j \neq Y, u_{j}(x, S) \geq 0$ only if $x \in I_{j}\left(p\left(u_{j}\right)\right)$ and $S=N$ with $I_{j} p\left(u_{j}\right) \cap I_{h} p\left(u_{h}\right)=\emptyset$ for any pair such that $j \notin Y$ and $h \neq j$. Consider $j \notin Y$ and profile $\left(u_{j}^{\prime}, u_{-j}\right)$ with $u_{j}^{\prime} \in U_{j}^{\text {shar }}$ such that $p\left(u_{j}^{\prime}\right)=c, u_{j}^{\prime}(x, S) \geq 0$ only if $x \in I_{j}^{\prime}(c) \subset I(c)$ and $u_{j}^{\prime}(c,\{j\})>0$. If agent $j \notin f^{G}\left(u_{j}^{\prime}, u_{-j}\right)$, then by outsider

\footnotetext{
${ }^{23}$ Noting that $j$ 's most preferred allocation has positive utility, this interval must have a non-empty interior.
} 
independence $f\left(u_{j}^{\prime}, u_{-j}\right)=(c, Y)$ and individual stability is violated. Therefore by strategy-proofness and individual stability $f\left(u_{j}^{\prime}, u_{-j}\right)=(z,(Y \cup j))$ with $z \in I_{j}^{\prime}(c)$. Since this must hold for any closed neighborhood of $c$, it follows $f\left(u_{j}^{\prime}, u_{-j}\right)=(c,(Y \cup j))$. Iterating for all agents $i \notin(Y \cup j)$, we obtain $f\left(u^{\prime}\right)=(c, N)$. By strategy-proofness it follows that $f\left(\tilde{u}^{c}\right)=(c, N)$.

Step 2: Let $f(u)=(c, Y)$ and $f(\dot{u})=(z, W)$. We show that $c=z$.

By Step 1, $f\left(\tilde{u}^{c}\right)=(c, N)$ and $f\left(\tilde{u}^{z}\right)=(z, N)$. Consider profile $\left(u_{1}^{z}, \tilde{u}_{-1}^{c}\right)$ where $p\left(u_{1}^{z}\right)=z$ and $u_{1}^{z}(x, S) \geq 0$ only if $x \in I_{1}(z)$ and such that $c \notin I_{1}(z)$. If $f^{L}\left(u_{1}^{z}, \tilde{u}_{-1}^{c}\right) \notin$ $I_{1}(z)$, then by individual stability agent $1 \notin f^{G}\left(u_{1}^{z}, \tilde{u}_{-1}^{c}\right)$ In this case strategy-proofness is violated at some profile since $f\left(u_{1}^{z}, \tilde{u}_{-1}^{z}\right)=f\left(\tilde{u}^{z}\right)=(z, N)$ and, for all $i \in N$, $\tilde{u}_{i}^{c}(z, N)>\tilde{u}_{i}^{c}(x, S)$ for all $S \neq N$, for all $x$. Since $f^{L}\left(u_{1}^{z}, \tilde{u}_{-1}^{c}\right) \in I_{1}(z)$ for all possible neighborhoods of $z$, then by strategy-proofness it must be $f\left(u_{1}^{z}, \tilde{u}_{-1}^{c}\right)=(z, N)$. Again by strategy-proofness $f\left(\tilde{u}_{1}^{z}, \tilde{u}_{-1}^{c}\right)=(z, N)$ and iterating for all $i \neq n, f\left(\tilde{u}_{1}^{z}, \ldots, \tilde{u}_{n-1}^{z}, \tilde{u}_{n}^{c}\right)=$ $f\left(\tilde{u}_{-n}^{z}, \tilde{u}_{n}^{c}\right)=(z, N)$. Consider profile $\left(\tilde{u}_{-n}^{z}, u_{n}^{c}\right)$ where $p\left(u_{n}^{c}\right)=c, u_{n}^{c}(c, S)>0$ for all $S$, and $u_{n}^{c}(x, S)<0$ if $x \notin I_{n}(c)$ with $z \notin I_{n}(c)$. By the same argument, we conclude that $f\left(\tilde{u}_{-n}^{z}, u_{n}^{c},\right)=(c, N)$ and by strategy-proofness $f\left(\tilde{u}_{-n}^{z}, \tilde{u}_{n}^{c}\right)=(c, N)$, which contradicts our previous conclusion that $f\left(\tilde{u}_{-n}^{z}, \tilde{u}_{n}^{c}\right)=(z, N)$.

Proof of Theorem 11: From Theorem 12 (proven below) we know that there is always just one agent assigned to consume and the level must be that agent's peak. We need only show that it must always be the same agent.

We first state an obvious fact that follows directly from strategy-proofness.

Fact 1: Let $f(u)=\left(p\left(u_{i}\right),\{i\}\right)$ for some $i \in I$. Then $f\left(\bar{u}_{i}, u_{-i}\right)=\left(p\left(\bar{u}_{i}\right),\{i\}\right)$ for all $\bar{u}_{i} \in U_{i}^{\text {cong }}$.

We now show that $f(u)=\left(p\left(u_{i}\right),\{i\}\right)$ implies $f(\bar{u})=\left(p\left(\bar{u}_{i}\right),\{i\}\right)$ for all $\bar{u} \in U^{\text {cong }}$.

Suppose the contrary. Without loss of generality, let $f(u)=\left(p\left(u_{1}\right),\{1\}\right)$ and $f(\bar{u})=\left(p\left(\bar{u}_{2}\right),\{2\}\right)$. By Fact $1 f\left(\bar{u}_{2}, u_{-2}\right) \neq\left(p\left(\bar{u}_{2}\right),\{2\}\right)$, as otherwise it would have to be that $f(u)=\left(p\left(\bar{u}_{2}\right),\{2\}\right)$. By outsider independence, $f\left(\bar{u}_{2}, u_{-2}\right)=\left(p\left(u_{1}\right),\{1\}\right)$. Similarly, $f\left(\bar{u}_{2}, \bar{u}_{3}, u_{-2,3}\right) \neq\left(p\left(\bar{u}_{3}\right),\{3\}\right)$ and so by outsider independence $f\left(\bar{u}_{2}, \bar{u}_{3}, u_{-2,3}\right)=$ $\left(p\left(u_{1}\right),\{1\}\right)$. Iterating this argument we reach the conclusion that $f\left(u_{1}, \bar{u}_{-1}\right)=\left(p\left(u_{1}\right),\{1\}\right)$. Therefore, by Fact $1 f(\bar{u})=\left(p\left(\bar{u}_{1}\right),\{1\}\right)$, which contradicts our supposition.

Proof of Theorem 12: We prove the theorem through a series of lemmas. 
Lemma 18 Let $f: U^{k} \rightarrow[0,1] \times 2^{N}$ be strategy-proof, Pareto efficient and outsider independent. Then for all $u \in U^{k}, k \geq\left|f^{G}(u)\right|$.

Proof of Lemma 18: Suppose the contrary. Then we can find $u \in U^{k}$ such that $f(u)=(a, Y)$ where $|Y|=q>k$ and $q$ is the minimal cardinality of any group in the range which is of size greater than $k$. Without loss of generality, assume $Y=(1, \ldots, q)$. By Lemma 16 we can assume, without loss of generality, that $p\left(u_{i}\right)=a, u_{i}(a, Y)>0$, $u_{i}(x, S) \geq 0$ only if $x \in I_{i}\left(p\left(u_{i}\right)\right)=I(a)$, and $q \geq|S| \geq k$, for all $i \in Y$. By Lemma 17 we assume that $p\left(u_{j}\right) \neq a, u_{j}(x, S) \geq 0$ only if $|S|=k$, and $x \in I_{j}\left(p\left(u_{j}\right)\right)$ with $I_{j}\left(p\left(u_{j}\right)\right) \cap I_{h}\left(p\left(u_{h}\right)\right)=\emptyset$ for all $h \neq j$.

Let us assume that $a \neq 1$, as the case where $a \neq 0$ is analogous. For any $i \in Y$, let $\bar{u}_{i}^{b} \in U_{i}^{k}$ be such that ${ }^{24} p\left(\bar{u}_{i}^{b}\right)=b \notin I(a)$, such that there does not exist any $j \neq i$ with $p\left(u_{j}\right) \in(a, b)$. Let $\bar{u}_{i}^{b}$ be such that $\bar{u}_{i}^{b}(x, S) \geq 0$ only if $q \geq|S| \geq k$ and $x \in I_{i}(b)=I(b)$, with $I(b) \cap I_{j}\left(p\left(u_{j}\right)\right)=\emptyset$ for all $j \notin Y$, and $\bar{u}_{i}^{b}(a, Y)>0$ (hence $a$ is interior to $I(b)$ ).

Let us consider profile $\left(\bar{u}_{1}^{b}, u_{-1}\right)$. Since $\bar{u}_{1}^{b}(f(u))>0$, by strategy-proofness and the conditions on preferences, it follows that $1 \in f^{G}\left(\bar{u}_{1}^{b}, u_{-1}\right),\left|f^{G}\left(\bar{u}_{1}^{b}, u_{-1}\right)\right| \geq k$, and $f^{L}\left(\bar{u}_{1}^{b}, u_{-1}\right)=y \in I(b)$. Suppose that either $f^{L}\left(\bar{u}_{1}^{b}, u_{-1}\right)=y$ such that $u_{i}(y, Y)<0$ for any $i \in Y$, or $\left|f^{G}\left(\bar{u}_{1}^{b}, u_{-1}\right)\right|<q$. By efficiency and the minimality of $Y$, it follows that $\left|f^{G}\left(\bar{u}_{1}^{b}, u_{-1}\right)\right|=k$. Let $u_{1}^{\prime \prime}$ be such that $p\left(u_{1}^{\prime \prime}\right)=a, u_{1}^{\prime \prime}(x, S) \geq 0$ if and only if $|S|=k, i \in S$ and $x \in I_{1}^{\prime \prime}(a)$; moreover let $I_{1}^{\prime \prime}(a)$ be such that $b \in \operatorname{int} I_{1}^{\prime \prime}(a)$ and $I_{1}^{\prime \prime}(a) \cap I\left(p\left(u_{j}\right)\right)=\emptyset$ for all $j \neq Y$. If $f\left(\bar{u}_{1}^{b}, u_{-1}\right)=\left(y, S_{k}\right)$ with $1 \in f^{G}\left(\bar{u}_{1}, u_{-1}\right)$, then by strategy-proofness, $1 \in f^{G}\left(u_{i}^{\prime \prime}, u_{-1}\right)$ and by efficiency $f\left(u_{1}^{\prime \prime}, u_{-1}\right)=\left(a, S_{k}\right)$. However, then strategy-proofness is violated at profile $u$ by agent 1 . Thus, consider the case where $f^{L}\left(\bar{u}_{1}^{b}, u_{-1}\right)=y$ with $u_{i}(y, Y) \geq 0$ for all $i \in Y$ and $\left|f^{G}\left(\bar{u}_{1}^{b}, u_{-1}\right)\right| \geq q$. By strategyproofness and the conditions on preferences it must be $\left|f^{G}\left(\bar{u}_{1}^{b}, u_{-1}\right)\right|=q$, hence by efficiency $f^{G}\left(\bar{u}_{1}^{b}, u_{-1}\right)=Y$. Suppose that $y \neq a$. Then consider $\dot{u}_{2}$ such that $p\left(\dot{u}_{2}\right)=a$ and $\dot{u}_{2}(x, S) \geq 0$ only if $x \in \dot{I}_{2}(a) \subset I(a)$ and $q \geq|S| \geq k$, with $y \notin \dot{I}_{2}(a)$ and $\dot{u}_{2}(a, Y)>0$. By strategy-proofness $f\left(\dot{u}_{2}, u_{-2}\right)=(a, Y)$. By the same reasoning as before it follows that $f\left(\bar{u}_{1}^{b}, \dot{u}_{2}, u_{-(1 \cup 2)}\right)=(z, Y)$ with $z \in \dot{I}_{2}(a)$, and strategy-proofness is violated at profile $\left(\bar{u}_{1}^{b}, u_{-1}\right)$ by agent 2 via $\dot{u}_{2}$, since $u_{2}(y, Y)<u_{2}(z, Y)$.

So, it follows that $f\left(\bar{u}_{1}^{b}, u_{-1}\right)=(a, Y)$. Repeating the same argument we conclude that

$$
f\left(\bar{u}_{1}^{b}, \ldots, \bar{u}_{q-1}^{b}, u_{q}, \ldots, u_{n}\right)=(a, Y)
$$

and by efficiency

$$
f\left(\bar{u}_{1}^{b}, \ldots, \bar{u}_{q-1}^{b}, \bar{u}_{q}^{b}, u_{q+1}, \ldots, u_{n}\right)=(b, Y)
$$

\footnotetext{
${ }^{24}$ The apex $b$ specifies the agent $i^{\prime} s$ peak according to the new preferences. We write the apex when the peak is different from the peak of the former preferences $u_{i}$.
} 
From now on we fix the utility functions of agents from $q+1$ to $n$, and simplify notation by writing only the first $q$ components of the utility profiles.

Consider $\hat{u}_{q}$ such that $p\left(\hat{u}_{q}\right)=a, \hat{u}_{q}(b, Y)>0$, and $\hat{u}_{q}(x, S) \geq 0$ only if $x \in \hat{I}_{q}(a)$ and $q \leq|S| \leq k$. Given (1), by strategy-proofness $q \in f^{G}\left(\left(\bar{u}_{1}^{b}, \ldots \bar{u}_{q-1}^{b}, \hat{u}_{q}\right)\right)$.

Suppose that $f^{L}\left(\left(\bar{u}_{1}^{b}, \ldots \bar{u}_{q-1}^{b}, \hat{u}_{q}\right)\right)=c$ with $c \neq a, c \in \hat{I}_{q}(a)$. Since $f\left(\bar{u}_{1}^{b}, \ldots, \bar{u}_{q-1}^{b}, u_{q}\right)=$ $(a, Y)$, then strategy-proofness implies $q<f^{G}\left(\left(\bar{u}_{1}^{b}, \ldots \bar{u}_{q-1}^{b}, \hat{u}_{q}\right)\right) \leq k$. By minimality of $Y$, it follows $\left|f^{G}\left(\left(\bar{u}_{1}^{b}, \ldots \bar{u}_{q-1}^{b}, \hat{u}_{q}\right)\right)\right|=k$. Consider, then, $\dot{u}_{q}^{b}$ such that $p\left(\dot{u}_{q}^{b}\right)=b$, and $\dot{u}_{q}^{b}\left(c, S_{k}\right)>\dot{u}_{q}^{b}(b, Y)$. By efficiency $f\left(\bar{u}_{1}^{b}, \ldots \bar{u}_{q-1}^{b}, \dot{u}_{q}^{b}\right)=\left(b, S_{k}\right)$ violating strategyproofness at profile $f\left(\bar{u}^{b}\right)$. It follows that $f^{L}\left(\left(\bar{u}_{1}^{b}, \ldots \bar{u}_{q-1}^{b}, \hat{u}_{q}\right)\right)=a$. By strategyproofness, given (1), we have that

$$
f\left(\bar{u}_{1}^{b}, \ldots \bar{u}_{q-1}^{b}, \hat{u}_{q}\right)=(a, Y) .
$$

Consider $\tilde{u}_{1}^{b}$ such that $p\left(\tilde{u}_{1}^{b}\right)=b$ and $\tilde{u}_{1}^{b}(x, S) \geq 0$ only if $x \in \tilde{I}_{1}(b)$ with $a \notin \tilde{I}_{1}(b)$, and $q \geq|S| \geq k$. By strategy-proofness, (2) implies that

$$
f\left(\tilde{u}_{1}^{b}, \bar{u}_{2}^{b}, \ldots, \bar{u}_{q}^{b}\right)=(b, Y) .
$$

Next, consider the profile $\left(\tilde{u}_{1}^{b}, \bar{u}_{2}^{b} \ldots, \bar{u}_{q-1}^{b}, \hat{u}_{q}\right)$. Since $\hat{u}_{q}(x, S) \geq 0$ only if $q \leq|S| \leq k$, and $\hat{u}_{q}(b, Y)>0$, strategy-proofness and (4) imply that agent $q \in f^{G}\left(\tilde{u}_{1}^{b}, \bar{u}_{2}^{b} \ldots, \bar{u}_{q-1}^{b}, \hat{u}_{q}\right)$ and $q \leq\left|f^{G}\left(\tilde{u}_{1}^{b}, \bar{u}_{2}^{b} \ldots, \bar{u}_{q-1}^{b}, \hat{u}_{q}\right)\right| \leq k$. If $f^{L}\left(\tilde{u}_{1}^{b}, \bar{u}_{2}^{b} \ldots, \bar{u}_{q-1}^{b}, \hat{u}_{q}\right) \in \tilde{I}_{1}(b)$ then strategyproofness is violated at (3) by agent 1 via $\tilde{u}_{1}^{b}$. Suppose, then, that $f^{L}\left(\tilde{u}_{1}^{b}, \bar{u}_{2}^{b} \ldots, \bar{u}_{q-1}^{b}, \hat{u}_{q}\right) \notin$ $\tilde{I}_{1}(b)$. Pareto efficiency implies that agent $1 \notin f^{G}\left(\tilde{u}_{1}^{b}, \bar{u}_{2}^{b} \ldots, \bar{u}_{q-1}^{b}, \hat{u}_{q}\right)$ and by the minimality of $Y,\left|f^{G}\left(\tilde{u}_{1}^{b}, \bar{u}_{2}^{b} \ldots, \bar{u}_{q-1}^{b}, \hat{u}_{q}\right)\right|=k$. Efficiency implies also that $f^{L}\left(\tilde{u}_{1}^{b}, \bar{u}_{2}^{b} \ldots, \bar{u}_{q-1}^{b}, \hat{u}_{q}\right)=$ $c \in[a, b)$. Consider $\dot{u}_{q}^{b}$ such that $p\left(\stackrel{\circ}{u}_{q}^{b}\right)=b, \stackrel{\circ}{u}_{q}^{b}\left(c, S_{k}\right)>0$, and $\stackrel{\circ}{u}_{q}^{b}(x, S)<0$ if $|S| \neq k$ or $x \notin[a, b]$. Given that $q \in f^{G}\left(\tilde{u}_{1}^{b}, \bar{u}_{2}^{b} \ldots, \bar{u}_{q-1}^{b}, \hat{u}_{q}\right)$, by strategy-proofness agent $q \in$ $f^{G}\left(\tilde{u}_{1}^{b}, \bar{u}_{2}^{b} \ldots, \bar{u}_{q-1}^{b}, \stackrel{\circ}{u}_{q}^{b}\right)$, and $\left|f^{G}\left(\tilde{u}_{1}^{b}, \bar{u}_{2}^{b} \ldots, \bar{u}_{q-1}^{b}, \dot{u}_{q}^{b}\right)\right|=k$. By efficiency $f\left(\tilde{u}_{1}^{b}, \bar{u}_{2}^{b} \ldots, \bar{u}_{q-1}^{b}, \stackrel{\leftrightarrow}{u}_{q}^{b}\right)=$ $\left(b, S_{k}\right)$, but strategy-proofness is violated at profile (4) by agent $q$ via $\dot{u}_{q}^{b}$. We have reached a contradiction and so $k \geq|Y|$.

Lemma 19 Let $f: U^{k} \rightarrow[0,1] \times 2^{N}$ be strategy-proof, efficient and outsider independent. Then for all $u \in U^{k},\left|f^{G}(u)\right| \geq k$.

Proof of Lemma 19: Suppose the contrary. Let $f(u)=(a, Y)$ with $|Y|=q<k$ where $q$ is the maximal cardinality of any group in the range that is smaller than $k$. Without loss of generality, assume that $Y=(1, \ldots, q)$. Also, by Lemma 16 we can assume that all 
agents $i \in Y$ have the same preferences with $p\left(u_{i}\right)=a, u_{i}(a, Y)>0$ and $u_{i}(x, S) \geq 0$ only if $x \in I_{i}\left(p\left(u_{i}\right)\right)=I(a)$. Let $I^{k}(a)=\left\{x \in I(a) \mid u_{i}\left(x, S_{k}\right) \geq u_{i}(a, Y)\right.$ foranyi $\left.\in Y\right\}$. By Lemma 17 we can assume that for all $j \notin Y, p\left(u_{j}\right) \neq a, u_{j}(x, S) \geq 0$ only if $|S|=k$ and $x \in I_{j} p\left(u_{j}\right)$ with $I_{j} p\left(u_{j}\right) \cap I_{i} p\left(u_{i}\right)=\emptyset$ for all $i \neq j$. Finally, without loss of generality, assume that $p\left(u_{q+1}\right)=b>a$ and there does not exist any agent $i \in N, i \neq q+1$ such that $p\left(u_{i}\right) \in(a, b]$.

For any $i \in Y$ let $u_{i}^{\prime}$ be such that $p\left(u_{i}^{\prime}\right)=a, u_{i}^{\prime}(x, S) \geq 0$ only if $x \in I_{i}^{\prime}(a)=I^{\prime}(a)$ with $I^{\prime}(a) \cap I_{j}\left(p\left(u_{j}\right)\right)=\emptyset$ for all $j \geq q+2$, and $k \geq|S| \geq q$, and $u_{i}^{\prime}\left(b, S_{q+1}\right)>u_{i}^{\prime}(a, Y)$. By efficiency and strategy-proofness $f^{G}\left(u_{1}^{\prime}, \ldots, u_{q}^{\prime}, u_{q+1}, \ldots, u_{n}\right) \supset\{1, \ldots, q+1\}$. Hence by the assumption of the maximality of $Y$ and strategy-proofness we have $f\left(u_{Y}^{\prime}, u_{-Y}\right)=\left(x, S_{k}\right)$ with $x \notin I^{k}(a)$. We fix the preferences of agents $q+2$ to $n$, and simplify notation by only writing the first $q+1$ components of each preference profile. Let $u_{q+1}^{\prime}$ be such that $p\left(u_{q+1}^{\prime}\right)=b u_{q+1}^{\prime}(x, S) \geq 0$ only if $x \in I_{q+1}^{\prime}(b)$, with, $I_{q+1}^{\prime}(b) \cap I_{j} p\left(u_{j}\right)=\emptyset$ for all $j \geq q+2$, and $k \geq|S|>q$. Let also $u_{q+1}^{\prime}$ be such that $u_{q+1}^{\prime}\left(a, S_{q+1}\right)>0$ (hence $\left.a \in \operatorname{int} I_{q+1}^{\prime}(b)\right)$. Then $f\left(u_{1}, \ldots u_{q}, u_{q+1}^{\prime}\right)=\left(x, S_{k}\right)$ with $x \neq b$. By strategy-proofness it follows that $f\left(u_{1}, \ldots u_{q}, u_{q+1}^{\prime}\right)=f\left(u_{1}^{\prime}, \ldots u_{q}^{\prime}, u_{q+1}^{\prime}\right)=f\left(u_{1}^{\prime}, \ldots u_{q}^{\prime}, u_{q+1}\right)=\left(c, S_{k}\right)$ with $c \in(a, b), c \notin I^{k}(a)$.

For any $i \in Y$, let $\tilde{u}_{i}$ be such that $p\left(\tilde{u}_{i}\right)=a, \tilde{u}_{i}(x, S) \geq 0$ only if $x \in \tilde{I}_{i}(a)=\tilde{I}(a)$ with $\tilde{I}(a) \subset I^{k}(a)$. Let $\bar{u}_{i}$ be such that $p\left(\bar{u}_{i}\right)=a, \bar{u}_{i}(a, Y)>\bar{u}_{i}\left(b, S_{k}\right)>0$ and $\bar{u}_{i}\left(x, S_{k}\right)<0$ for all $x \notin \bar{I}_{i}(a)=\bar{I}(a)$ with $\bar{I}(a) \cap I_{j}\left(p\left(u_{j}\right)\right)=\emptyset$ for all $j \geq q+2$. Let $\bar{I}^{k}(a)=\left\{x \in \bar{I}(a) \mid \bar{u}_{i}\left(x, S_{k}\right) \geq \bar{u}_{i}(a, Y)\right.$ foranyi $\left.\in Y\right\}$. Let $\bar{u}_{q+1}$ be such that $p\left(\bar{u}_{q+1}\right)=$ $b, \bar{u}_{q+1}(b,\{q+1\})>\bar{u}_{q+1}\left(x, S_{k}\right)$ for all $x \notin[b-\varepsilon, b+\varepsilon]$ with $(b-\varepsilon) \notin \bar{I}^{k}(a)$, and $\bar{u}_{q+1}\left(a, S_{k}\right)>0$, and $\bar{u}_{q+1}\left(x, S_{k}\right)<0$ for all $x \notin \bar{I}_{q+1}(b)$ with $\bar{I}_{q+1}(b) \cap I_{j}\left(p\left(u_{j}\right)\right)=\emptyset$ for all $j \geq q+2$.

First, consider profile $\left(u_{1}^{\prime}, u_{2}^{\prime} \ldots u_{q}^{\prime}, \bar{u}_{q+1}\right)$. Since $\bar{u}_{q+1}(b,\{q+1\})>\bar{u}_{q+1}\left(a, S_{k}\right)>0$ and $u_{i}^{\prime}\left(b, S_{q+1}\right)>0$ for all $i \in\{1, \ldots, q\}$, then by efficiency $\{1, \ldots, q+1\} \subset f^{G}\left(u_{1}^{\prime}, u_{2}^{\prime} \ldots u_{q}^{\prime}, \bar{u}_{q+1}\right)$. Since $f\left(u^{\prime}\right)=\left(c, S_{k}\right)$ then by strategy-proofness and by maximality of $Y f\left(u_{1}^{\prime}, u_{2}^{\prime} \ldots u_{q}^{\prime}, \bar{u}_{q+1}\right)=$ $\left(c, S_{k}\right)$. Next, consider profile $\left(\tilde{u}_{1}, u_{2}^{\prime} \ldots u_{q}^{\prime}, \bar{u}_{q+1}\right)$.

We argue that agent $q+1 \in f^{G}\left(\tilde{u}_{1}, u_{2}^{\prime} \ldots u_{q}^{\prime}, \bar{u}_{q+1}\right)$. Suppose not. Consider $u_{q+1}^{\prime \prime}$ such that $p\left(u_{q+1}^{\prime \prime}\right)=a$ and $u_{q+1}^{\prime \prime}(x, S) \geq 0$ if and only if $k \geq|S| \geq q$ and $x \in I_{q+1}^{\prime \prime}(a) \subset I(a)$. By outsider independence if agent $q+1 \notin f^{G}\left(u_{1}, . ., u_{q}, u_{q+1}^{\prime \prime}\right)$ then $f\left(u_{1}, . ., u_{q}, u_{q+1}^{\prime \prime}\right)=$ $f(u)$ and efficiency is violated. Therefore agent $q+1 \in f^{G}\left(u_{1}, . ., u_{q}, u_{q+1}^{\prime \prime}\right)$ and by strategy-proofness and the maximality of $Y, f\left(u_{1}, . ., u_{q}, u_{q+1}^{\prime \prime}\right)=\left(a, S_{k}\right)$. It follows by strategy-proofness that $f\left(u_{1}^{\prime}, . ., u_{q}^{\prime}, u_{q+1}^{\prime \prime}\right)=\left(a, S_{k}\right)$ and, consequently $f\left(\tilde{u}_{1}, u_{2}^{\prime} . ., u_{q}^{\prime}, u_{q+1}^{\prime \prime}\right)=$ $\left(a, S_{k}\right)$. Since $\bar{u}_{q+1}\left(a, S_{k}\right)>0$, by strategy-proofness it follows that agent $q+1 \in$ $f^{G}\left(\tilde{u}_{1}, u_{2}^{\prime} . ., u_{q}^{\prime}, \bar{u}_{q+1}\right)$. 
Now we argue that, $f^{L}\left(\tilde{u}_{1}, u_{2}^{\prime} \ldots u_{q}^{\prime}, \bar{u}_{q+1}\right) \notin \tilde{I}(a)$. In fact, if $f^{L}\left(\tilde{u}_{1}, u_{2}^{\prime} \ldots u_{q}^{\prime}, \bar{u}_{q+1}\right) \in$ $\tilde{I}(a)$ then by efficiency and the maximality of $Y$, it follows that $\left|f^{G}\left(\tilde{u}_{1}, u_{2}^{\prime} \ldots u_{q}^{\prime}, \bar{u}_{q+1}\right)\right|=k$ and strategy-proofness is violated by agent 1 at profile $\left(u_{1}^{\prime}, u_{2}^{\prime} \ldots u_{q}^{\prime}, \bar{u}_{q+1}\right)$ via $\tilde{u}_{1}$. Therefore $f^{L}\left(\tilde{u}_{1}, u_{2}^{\prime} . ., u_{q}^{\prime}, \bar{u}_{q+1}\right) \in\left(\max \tilde{I}(a), \max \bar{I}_{q+1}(b)\right]$. Next, consider the profile $\left(\tilde{u}_{1}, \tilde{u}_{2}, u_{3}^{\prime} . ., u_{q}^{\prime}, \bar{u}_{q+1}\right)$. If agent $2 \notin f^{G}\left(\tilde{u}_{1}, u_{2}^{\prime} . ., u_{q}^{\prime}, \bar{u}_{q+1}\right)$, then by outsider independence $f\left(\tilde{u}_{1}, u_{2}^{\prime} \ldots, u_{q}^{\prime}, \bar{u}_{q+1}\right)=$ $f\left(\tilde{u}_{1}, \tilde{u}_{2}, u_{3}^{\prime} . ., u_{q}^{\prime}, \bar{u}_{q+1}\right)$. If agent $2 \in f^{G}\left(\tilde{u}_{1}, u_{2}^{\prime} . ., u_{q}^{\prime}, \bar{u}_{q+1}\right)$ we may use the same argument as given above to prove that agent $q+1 \in f^{G}\left(\tilde{u}_{1}, \tilde{u}_{2}, u_{3}^{\prime} . ., u_{q}^{\prime}, \bar{u}_{q+1}\right)$ and $f^{L}\left(\tilde{u}_{1}, \tilde{u}_{2} . ., u_{q}^{\prime}, \bar{u}_{q+1}\right) \in$ $\left(\max \tilde{I}(a), \max \bar{I}_{q+1}(b)\right]$. Iterating this argument, we conclude that agent $q+1 \in$ $f^{G}\left(\tilde{u}_{1}, \tilde{u}_{2} \ldots \tilde{u}_{q}, \bar{u}_{q+1}\right)$. Since at the allocation $f\left(\tilde{u}_{1}, \tilde{u}_{2} \ldots \tilde{u}_{q}, \bar{u}_{q+1}\right)$ the utility level of all agents in $f^{G}\left(\tilde{u}_{1}, \tilde{u}_{2} \ldots \tilde{u}_{q}, \bar{u}_{q+1}\right)$ is negative, except the utility level of agent $q+1$, and $\bar{u}_{q+1}(b,\{q+1\})>0$, it follows that any efficient allocation provides $f^{L}\left(\tilde{u}_{1}, \tilde{u}_{2} \ldots \tilde{u}_{q}, \bar{u}_{q+1}\right) \in$ $[b-\varepsilon, b+\varepsilon]$. Let $\tilde{u}_{q+1}$ be such that $p\left(\tilde{u}_{q+1}\right)=b, \tilde{u}_{q+1}(x, S) \geq 0$ only if $x \in \tilde{I}_{q+1}(b)=$ $\tilde{I}(b)$, with $c \notin \tilde{I}(b)$, and $\tilde{u}_{q+1}(x,\{q+1\})>0$ for all $x \in[b-\varepsilon, b+\varepsilon]$. Moreover let $\tilde{u}_{q+1}$ be such that for any $x<y<b(x>y>b) \tilde{u}_{q+1}(x, S) \geq \tilde{u}_{q+1}\left(y, S^{\prime}\right)$ implies $\bar{u}_{q+1}(x, S)>\bar{u}_{q+1}\left(y, S^{\prime}\right)$. By strategy-proofness

$$
f^{L}(\tilde{u}) \in[b-\varepsilon, b+\varepsilon]
$$

Consider now the profile $f\left(\bar{u}_{1}, u_{2}^{\prime}, \ldots, u_{q+1}^{\prime}\right)$. As we previously showed $f\left(u_{1}^{\prime}, u_{2}^{\prime} \ldots u_{q}^{\prime}, u_{q+1}^{\prime}\right)=$ $\left(c, S_{k}\right)$. Given that $\bar{u}_{1}\left(c, S_{k}\right)>0$, it follows from strategy-proofness that agent $1 \in$ $f^{G}\left(\bar{u}_{1}, u_{2}^{\prime} \ldots u_{q}^{\prime}, u_{q+1}^{\prime}\right)$. Since $u_{q+1}^{\prime}\left(b, S_{q+1}\right)>0$, it follows from strategy-proofness and the maximality of $Y$ that $f\left(\bar{u}_{1}, u_{2}^{\prime} \ldots u_{q}^{\prime}, u_{q+1}^{\prime}\right)=\left(c, S_{k}\right)$. Iterating the same argument we conclude that $f\left(\bar{u}_{1}, \ldots \bar{u}_{q}, u_{q+1}^{\prime}\right)=\left(c, S_{k}\right)$. Now change agent $q+1$ 's preferences to $\tilde{u}_{q+1}$. By efficiency and the maximality of $Y$, if $f^{L}\left(\bar{u}_{1}, \ldots \bar{u}_{q}, \tilde{u}_{q+1}\right) \in \tilde{I}(b)$, since $\bar{u}_{i}\left(b, S_{k}\right)>0$ for all $i \in Y$, then $f^{G}\left|\left(\bar{u}_{1}, \ldots \bar{u}_{q}, \tilde{u}_{q+1}\right)\right|=k$ and strategy-proofness is violated at profile $\left(\bar{u}_{1}, \ldots \bar{u}_{q}, u_{q+1}^{\prime}\right)$ by agent $q+1$ via $\tilde{u}_{q+1}$. Therefore $f^{L}\left(\bar{u}_{1}, \ldots \bar{u}_{q}, \tilde{u}_{q+1}\right) \notin \tilde{I}(b)$ and $\tilde{u}_{q+1}\left(f\left(\bar{u}_{1}, \ldots \bar{u}_{q}, \tilde{u}_{q+1}\right)\right)<0$. Since $\bar{u}_{i}(a, Y)>\bar{u}_{i}\left(b, S_{k}\right)$ for all $i \in Y$, by efficiency, $f^{L}\left(\bar{u}_{1}, \ldots \bar{u}_{q}, \tilde{u}_{q+1}\right) \in I^{k}(a)$ and by strategy-proofness agent $q+1 \notin f^{G}\left(\bar{u}_{1}, \ldots \bar{u}_{q}, \tilde{u}_{q+1}\right)$, otherwhise he would announce $u_{q+1}^{\prime}$. It follows from strategy-proofness that $f\left(\tilde{u}_{1}, \ldots \tilde{u}_{q}, \tilde{u}_{q+1}\right)=$ $(a, S)$ with $q+1 \notin S$, which contradicts the previous conclusion in equation (5).

Lemma 20 Let $f: U^{k} \rightarrow[0,1] \times 2^{N}$ be strategy-proof, efficient and outsider independent. Then for each $u \in U^{k}$, there exists some $i \in f^{G}(u)$ with $p\left(u_{i}\right)=f^{L}(u)$.

Proof of Lemma 20: Suppose the contrary for some $u$, and let $f(u)=(b, Y)$, with $p\left(u_{i}\right) \neq b$ for all $i \in Y$. Using the same argument as in the proof of Lemma 17, we assume without loss of generality that $p\left(u_{j}\right) \neq b$ for all $j \notin Y$. From now on we do not specify the cardinality of the assigned group since we have already proven that it must be $k$ at all $u \in U^{k}$. The following claim is useful. 
Claim: If $f(u)=(a, S)$, with $i \in S$ and $p\left(u_{i}\right)<a$, then for all $x \in\left(p\left(u_{i}\right), a\right)$ there exists $u_{i}^{\prime}$ such that $p\left(u_{i}^{\prime}\right)=x$ and $f\left(u_{i}^{\prime}, u_{-i}\right)=\left(a, S^{\prime}\right)$ with $i \in S^{\prime}$ (and the same holds for the case where $\left.p\left(u_{i}\right)>a\right)$.

Proof of the Claim: Consider, in fact, any $u_{i}^{\prime \prime}$ such that $p\left(u_{i}^{\prime \prime}\right)=x$ with $x \in$ $\left(p\left(u_{i}\right), a\right)$, and $u_{i}^{\prime \prime}(a, S)>0$. By strategy-proofness it follows that $i \in f^{G}\left(u_{i}^{\prime \prime}, u_{-i}\right)$. Then $f^{L}\left(u_{i}^{\prime \prime}, u_{-i}\right) \neq a$ implies by strategy-proofness $f^{L}\left(u_{i}^{\prime \prime}, u_{-i}\right)=y$ with $y<p\left(u_{i}\right)$ and $u_{i}^{\prime \prime}(y, S) \geq u_{i}^{\prime \prime}(a, S)$. Consider then $u_{i}^{\prime}$ such that $p\left(u_{i}^{\prime}\right)=p\left(u_{i}^{\prime \prime}\right), u_{i}^{\prime}(a, S)>0$, but $u_{i}^{\prime}(y, S)<0$. Then by strategy-proofness $f\left(u_{i}^{\prime}, u_{-i}\right)=\left(a, S^{\prime}\right)$ with $i \in S^{\prime}$.

Suppose that $Y=\{1, \ldots, k\}$, and by the claim that $p\left(u_{1}\right)=\ldots p\left(u_{q}\right)=a<b$, $p\left(u_{q+1}\right)=\ldots=p\left(u_{k}\right)=c>b$, and for all $j \notin Y, p\left(u_{j}\right) \notin[a, c]$. Let $A=\{1, \ldots, q\}$ and $C=\{q+1, \ldots, k\}$.let assume that for all $i \in A(C) u_{i}(x, S) \geq 0$ only if $x \in$ $I_{i}\left(p\left(u_{i}\right)\right)=I(a)(=I(c))$ such that $[a, c] \subset I_{i}\left(p\left(u_{i}\right)\right)$, while for all $j \notin Y, u_{j}(x, S) \geq 0$ only if $x \in I_{j}\left(p\left(u_{j}\right)\right)$ and $|S|=k$, with $I_{j}\left(p\left(u_{j}\right)\right) \cap I_{i}\left(p\left(u_{i}\right)\right)=\emptyset$ for any pair such that $j \notin Y, i \in N$. . Consider any $i \in Y$. Let $\bar{u}_{i}$ be such that $p\left(\bar{u}_{i}\right)=p\left(u_{i}\right)$ and $\bar{u}_{i}(x, S) \geq 0$ only if $x \in \bar{I}_{i}\left(p\left(u_{i}\right)\right)$, where $\bar{I}_{i}\left(p\left(u_{i}\right)\right) \subset I_{i}\left(p\left(u_{i}\right)\right), b \notin \bar{I}_{i}\left(p\left(u_{i}\right)\right)$ and such that for all $x \in \bar{I}_{i}\left(p\left(u_{i}\right)\right) u_{i}(x, S)>u_{i}(b, S)$. Consider preference profile $\left(\bar{u}_{1}, u_{-1}\right)$. By efficiency if $f^{L}\left(\left(\bar{u}_{1}, u_{-1}\right)\right)=x$ for any $x \in \bar{I}_{i}\left(p\left(u_{i}\right)\right)$, then $f^{G}\left(\left(\bar{u}_{1}, u_{-1}\right)\right)=Y$, which violates strategyproofness at profile $u$. So, consider the following remaining cases. We show that each cannot hold.

CASE 1: $f\left(\bar{u}_{1}, u_{-1}\right)=\left(y, S^{\prime}\right)$ with $y<a$ or $y>\max I(c)$.

Consider any $i \in C$. Let $u_{i}^{\prime}$ be such that $p\left(u_{i}^{\prime}\right)=a$ and $u_{i}^{\prime}(x, S) \geq 0$ with $i \in S$ only if $x \in I(a)$ with $u_{i}^{\prime}(b, Y)>0$. By strategy-proofness and efficiency $f\left(u_{C}^{\prime}, u_{-C}\right)=(a, Y)$. By strategy-proofness $f\left(\bar{u}_{1}, u_{C}^{\prime}, u_{-(C \cup 1)}\right)=(a, Y)$. Therefore since $u_{i}\left(y, S^{\prime}\right)<u_{i}(a, Y)$ for all $i \in C$, strategy-proofness is violated at some utility profile.

CASE 2: $f\left(\bar{u}_{1}, u_{-1}\right)=\left(y, S^{\prime}\right)$ with $y \in\left(\max \bar{I}_{1}(a), b\right)$.

Agent $1 \notin S^{\prime}$, otherwhise strategy-proofness is violated at profile $u$ by agent 1 via $\bar{u}_{1}$. Consider then $u_{1}^{\prime}$ such that $p\left(u_{1}^{\prime}\right)=d$ with $a<d<y$ and $u_{1}^{\prime}\left(x, S^{\prime}\right) \geq 0$ if and only if agent $x \in[a, y]$ with $1 \in S^{\prime}$. By outsider independence if agent $1 \notin f\left(u_{1}^{\prime}, u_{-1}\right)$, then $f\left(u_{1}^{\prime}, u_{-1}\right)=f\left(\bar{u}_{1}, u_{-1}\right)$ and efficiency is violated. Therefore agent $1 \in f\left(u_{1}^{\prime}, u_{-1}\right)$ and $f^{L}\left(u_{1}^{\prime}, u_{-1}\right) \in[a, y]$ and strategy-proofness is violated at profile $u$ by agent 1 via $u_{1}^{\prime}$.

CASE 3: $f\left(\bar{u}_{1}, u_{-1}\right)=\left(y, S^{\prime}\right)$ with $y \in[b, \max I(c)]$.

By efficiency $C \subset S^{\prime}$. Change one by one preferences of all agents $i \in A$. Then $f\left(\bar{u}_{A}, u_{-A}\right)=\left(z, S^{\prime \prime}\right)$ with $z \in[b, \max I(c)]$, otherwise Case 1 or 2 above apply at some profile. By efficiency $C \subset S^{\prime \prime}$. Suppose that $z \neq c$. For $i \in C$, find preferences such that $p\left(u_{i}^{\prime}\right)=c, u_{i}^{\prime}(z, S)>0$ but $u_{i}^{\prime}\left(c, S_{k-q}\right)>u_{i}^{\prime}(z, S)$. By efficiency $f^{L}\left(\bar{u}_{A}, u_{C}^{\prime}, u_{-(A \cup C)}\right)=c$ 
with $C \subset f^{G}\left(\bar{u}_{A}, u_{C}^{\prime}, u_{-(A \cup C)}\right)$, since the outcome $(c, C)$ Pareto dominates $\left(z, S^{\prime \prime}\right)$. It follows that strategy-proofness is violated at some profile, since $\left|f^{G}\left(\bar{u}_{A}, u_{C}^{\prime}, u_{-(A \cup C)}\right)\right|=k$ by Lemmas 18 and 19. Therefore $f\left(\bar{u}_{A}, u_{-A}\right)=\left(c, S^{\prime \prime}\right)$ with $C \subset S^{\prime \prime}$ and by strategyproofness $f\left(\bar{u}_{A \cup C}, u_{-(A \cup C)}\right)=\left(c, S^{\prime \prime}\right)$.

A similar argument, starting looking at profile $\left(\bar{u}_{k}, u_{-k}\right)$ provides that $f\left(\bar{u}_{A \cup C}, u_{-(A \cup C)}\right)=$ $(a, S)$ reaching a contradiction.

Lemma 21 Let $f: U^{k} \rightarrow[0,1] \times 2^{N}$ be strategy-proof, efficient and outsider independent. If $u \in U^{k}$ and $u^{\prime} \in U^{k}$ are such that $p\left(u_{i}\right)=p\left(u_{i}^{\prime}\right)$ for all $i$, then $f^{L}(u)=f^{L}\left(u^{\prime}\right)$.

Proof of Lemma 21: From the previous lemmas, we know that $f$ picks the peak of some agent and selects a group of size $k$. This is taken as given in the proof that follows.

Let $\widehat{U}^{k} \subset U^{k}$ be the set of profiles for which $0 \neq u_{i}\left(p\left(u_{j}\right), S\right) \neq u_{i}\left(p\left(u_{i}\right), S\right)$ whenever $i \in S$ and $j \neq i$.

We establish the Lemma in the following steps.

Step 1: If $u \in \widehat{U}^{k}$ and $\widetilde{u}_{i}=u_{i}+M$ for some large $M$ (so that $\widetilde{u}_{i}(x, S)>0$ for any $x$ whenever $i \in S)$, then $f^{L}(\widetilde{u})=f^{L}(u)$.

Let $f(\widetilde{u})=(a, S)$. By strategy-proofness and outsider independence it follows that $f\left(\widetilde{u}_{S}, u_{-S}\right)=(a, S)$. Consider $j \in S$. Let us show that $f^{L}\left(\widetilde{u}_{S \backslash j}, u_{-S \cup j}\right)=a$. Iterating the same logic then establishes Step 1. First consider the case where $u_{j}(a, S)>0$. It follows from strategy-proofness that $j \in f^{G}\left(\widetilde{u}_{S \backslash j}, u_{-S \cup j}\right)$. Given that on $\widehat{U}^{k} u_{j}$ is not indifferent over any of the peaks of the agents, that $f^{L}$ picks a peak, and that $u_{j}$ and $\widetilde{u}_{j}$ have the same ranking of levels, it follows from strategy-proofness that $f^{L}\left(\widetilde{u}_{S \backslash j}, u_{-S \cup j}\right)=a$. Next, consider the case where $0 \geq u_{j}(a, S)$. Find $i$ such that $a=p\left(\widetilde{u}_{i}\right)=p\left(u_{i}\right)$. For any $\varepsilon>0$, define $\widehat{u}_{i}^{e}$ to be a shift of $\widetilde{u}_{i}$ where $p\left(\widehat{u}_{i}^{e}\right)=(1-\varepsilon) p\left(\widetilde{u}_{i}\right)+\varepsilon p\left(u_{j}\right)$. It follows from strategy-proofness, the fact that $f^{L}$ picks a peak, and the continuity of $\widehat{u}_{i}^{e}$ that for small enough $\varepsilon$ (and taking it so that the peak does not overlap with another peak)

$$
f^{L}\left(\widehat{u}_{i}^{e}, \widetilde{u}_{S \backslash i}, u_{-S}\right)=p\left(\widehat{u}_{i}^{e}\right) .
$$

Define $\widehat{u}_{j}^{e}$ so that the ranking over levels is the same as under $\widetilde{u}_{j}$, but so that $\widehat{u}_{j}^{e}(a, S)<0$ and $\widehat{u}_{j}\left(p\left(\widehat{u}_{i}^{e}\right)\right)>0$. Then it follows that

$$
f^{L}\left(\widehat{u}_{i}^{e}, \widehat{u}_{j}^{e}, \widetilde{u}_{S \backslash i, j}, u_{-S}\right)=p\left(\widehat{u}_{i}^{e}\right) .
$$

This follows from strategy-proofness and (6) if $j \in f^{L}\left(\widehat{u}_{i}^{e}, \widetilde{u}_{S \backslash i}, u_{-S}\right)$, and from outsider independence and strategy-proofness otherwise. Then it must be by strategy-proofness (via agent $i$ ), (7), and the fact that $f^{L}$ picks a peak, that for small enough $\varepsilon$

$$
f^{L}\left(\widehat{u}_{j}^{e}, \widetilde{u}_{S \backslash j}, u_{-S}\right)=a .
$$


Then if $j \in f^{G}\left(\widehat{u}_{j}^{e}, \widetilde{u}_{S \backslash j}, u_{-S}\right)$ it must be by strategy-proofness (and the fact that $\widehat{u}_{j}^{e}$ and $u_{j}$ have the same ranking over levels) that $f^{L}\left(\widetilde{u}_{S \backslash j}, u_{-S \cup j}\right)=a$. So consider the case where $j \notin f^{G}\left(\widehat{u}_{j}^{e}, \widetilde{u}_{S \backslash j}, u_{-S}\right)$. If also, $j \notin f^{G}\left(\widetilde{u}_{S \backslash j}, u_{-S \cup j}\right)$, then outsider independence establishes the conclusion of the step. If $j \in f^{G}\left(\widetilde{u}_{S \backslash j}, u_{-S \cup j}\right)$, then if $f^{L}\left(\widetilde{u}_{S \backslash j}, u_{-S \cup j}\right) \neq a$, then it must be that $u_{j}\left(f\left(\widetilde{u}_{S \backslash j}, u_{-S \cup j}\right)\right)>0$, which implies that $\widehat{u}_{j}^{e}\left(f\left(\widetilde{u}_{S \backslash j}, u_{-S \cup j}\right)\right)>0$ for small $\varepsilon$, which contradicts strategy-proofness and (8).

Step 2: If $u \in \widehat{U}^{k}$ and $u^{\prime} \in \widehat{U}^{k}$ are such that $p\left(u_{i}\right)=p\left(u_{i}^{\prime}\right)$ for all $i$, then $f^{L}(u)=f^{L}\left(u^{\prime}\right)$.

From step 1, we can concentrate on the case where $u_{i}(x, S)>0$ for all $x$ when $i \in S$ and similarly for $u_{i}^{\prime}$. Let $f(u)=(a, S)$, where $a=p\left(u_{i}\right)$. By strategy-proofness and outsider independence it follows that $f\left(u_{S}, u_{-S}^{\prime}\right)=(a, S)$. It then easily follows that $f^{L}\left(u_{S \backslash i}, u_{-S \cup i}^{\prime}\right)=a$. Next, change $u_{j}$ to $u_{j}^{\prime}$ for some $j \in S \backslash i$. Let us show that the outcome must still be $a$. Then iteration of this argument for further agents in $S$ establishes the step. Suppose to the contrary that $f^{L}\left(u_{S \backslash i, j}, u_{-S \cup i, j}^{\prime}\right)=b \neq a$. By outsider independence, it must be that $j \in f^{G}\left(u_{S \backslash i}, u_{-S \cup i}^{\prime}\right)$ or $f^{G}\left(u_{S \backslash i, j}, u_{-S \cup i, j}^{\prime}\right)$, and so by strategy-proofness $j$ must be in both groups. Also by strategy-proofness, it must be that $b \neq p\left(u_{j}\right)=p\left(u_{j}^{\prime}\right) \neq a$. In particular, it follows from strategy-proofness that $b$ must lie on one side of $p\left(u_{j}\right)$ and $a$ on the other side. For any $\varepsilon>0$, consider $u_{j}^{\varepsilon}$ with $p\left(u_{j}^{\varepsilon}\right)=p\left(u_{j}\right)$ and such that $u_{j}^{\varepsilon}(b)>u_{j}^{\varepsilon}(a)$, but $u_{j}^{\varepsilon}((1-\varepsilon) a+\varepsilon b)>u_{j}^{\varepsilon}(b)$, and where $u_{j}^{\varepsilon}(x, T)>0$ whenever $j \in T$. It follows from strategy-proofness that

$$
f^{L}\left(u_{j}^{\varepsilon}, u_{S \backslash i, j}, u_{-S \cup i}^{\prime}\right)=b .
$$

Let $u_{i}^{\varepsilon}$ be such that $p\left(u_{i}^{\varepsilon}\right)=(1-\varepsilon) a+\varepsilon b$ and $u_{i}^{\varepsilon}(x, T)>0$ whenever $i \in T$, and such that $u_{i}^{\varepsilon}(a)>u_{i}^{\varepsilon}(b),{ }^{25}$ but any peak of some other agent $h \neq i$ that lies on the opposite side of $a$ from $b$ is worse than $b$ under $u_{i}^{\varepsilon}$. For small $\varepsilon$, since $f^{L}\left(u_{S}, u_{-S}^{\prime}\right)=a$, then by strategy-proofness, continuity of preferences and the fact that $f^{L}$ always picks a peak of an agent, it follows that

$$
f^{L}\left(u_{i}^{\varepsilon}, u_{S \backslash i}, u_{-S}^{\prime}\right)=(1-\varepsilon) a+\varepsilon b,
$$

and that $i \in f^{G}\left(u_{i}^{\varepsilon}, u_{S \backslash i}, u_{-S}^{\prime}\right)$. By strategy-proofness (for $i$ ) and outsider independence, and (9) it follows that

$$
f^{L}\left(u_{i}^{\varepsilon}, u_{j}^{\varepsilon}, u_{S \backslash i, j}, u_{-S}^{\prime}\right)=b .
$$

[Under strategy-proofness, the only possibility is to change to the other side of $a$ but then it would have to be the peak of some other agent which is worse for $i$ than $b$.] However, now (10) and (11) contradict strategy-proofness for $j$ via a manipulation at $u_{j}^{\varepsilon}$.

Step 3: If $u \in U^{k}$ and $u^{\prime} \in U^{k}$ are such that $p\left(u_{i}\right)=p\left(u_{i}^{\prime}\right)$ for all $i$, then $f^{L}(u)=f^{L}\left(u^{\prime}\right)$.

The proof of this step follows from finding $u^{\varepsilon} \in \widehat{U}^{k}$ arbitrarily "close" to $u$ and $u^{\varepsilon^{\prime}} \in \widehat{U}^{k}$ arbitrarily "close" to $u^{\prime}$ such that the peaks of $u^{\varepsilon}$ and $u^{\varepsilon^{\prime}}$ coincide. From Step

\footnotetext{
${ }^{25}$ Since we have established that the size of the group must be fixed at $k$, we write the utility function as a function of the location; denoting the utility that would be obtained if $i$ were in the group of size $k$.
} 
2 we know that $f^{L}\left(u^{\varepsilon}\right)=f^{L}\left(u^{\varepsilon^{\prime}}\right)$. Showing that careful selection of these profiles leads to outcomes arbitrarily close to $f^{L}(u)$ and $f^{L}\left(u^{\prime}\right)$, respectively, provides the result.

Consider any $\varepsilon>0$. We identify $u_{i}^{\varepsilon}$ inductively on the agents. First consider agent 1. If $1 \notin f^{G}(u)$ find $u_{1}^{\varepsilon}$ such that $u_{1}^{\varepsilon}$ has a strict ranking over the (distinct) peaks of other agents, $\max _{x, S}\left|u_{1}(x, S)-u_{1}^{\varepsilon}(x, S)\right|<\varepsilon$, and $u_{1}^{\varepsilon}(x, S)<u_{1}(x, S)$ whenever $1 \in$ $S$. It follows from outsider independence that $f\left(u_{-1}, u_{1}^{\varepsilon}\right)=f(u)$. If $1 \in f^{G}(u)$, then find $u_{1}^{\varepsilon}$ such that $u_{1}^{\varepsilon}$ has a strict ranking over the (distinct) peaks of the other agents, $\max _{x, S}\left|u_{1}(x, S)-u_{1}^{\varepsilon}(x, S)\right|<\varepsilon$, and $\left\{x \mid u_{1}^{\varepsilon}\left(x, f^{G}(u)\right)>u_{1}^{\varepsilon}(f(u))\right\}$ is a strict subset of $\left\{x \mid u_{1}\left(x, f^{G}(u)\right)>u_{1}^{\varepsilon}(f(u))\right\}$, and $u_{1}^{\varepsilon}(f(u))>0$ if $u_{1}(f(u)) \geq 0$. By strategy-proofness, it must be that $1 \in f^{G}\left(u_{-1}, u_{1}^{\varepsilon}\right)$. Then the fact that $\left\{x \mid u_{1}^{\varepsilon}\left(x, f^{G}(u)\right)>u_{1}^{\varepsilon}(f(u))\right\}$ is a strict subset of $\left\{x \mid u_{1}\left(x, f^{G}(u)\right)>u_{1}^{\varepsilon}(f(u))\right\}$ and strategy-proofness imply that either $f^{L}\left(u_{-1}, u_{1}^{\varepsilon}\right)=f^{L}(u)$ or that $f^{L}\left(u_{-1}, u_{1}^{\varepsilon}\right)$ is arbitrarily near $f^{L}(u)$ for small enough $\varepsilon$, (as if it is not the first case then it must have been that $\left.f^{L}(u)=p\left(u_{1}\right)\right)$.

Then inducting and making similar changes for $i>1$ (taking care when changing $i$ 's peak not to induce indifference over peaks for any $j<i$ ) provides that $f^{L}\left(u^{\varepsilon}\right)$ can be made arbitrarily close to $f^{L}(u)$. When selecting $u^{\varepsilon^{\prime}}$, choose it to coincide with the peaks of $u^{\varepsilon}$.

The Lemmas above establish Theorem 12.

Proof of Theorem 14: We show that any individually stable rule cannot be strategyproof. Let $S_{q}$ denote any group with cardinality equal to $q$. Let $u \in U^{\mathrm{k}}$ with $k<n$ be such that for all $i \in N$

- $u_{i}(x, S)>0$ for all $x \in[0,1]$ and for all $S$ such that $|S| \leq k$, with $i \in S$, and

- $u_{i}\left(x, S^{\prime}\right)<0$ for all $x \in[0,1]$ and for all $S^{\prime}$ such that $\left|S^{\prime}\right|>k$, with $i \in S^{\prime}$.

By individual stability $f(u)=\left(y, S_{k}\right)$ for some $y \in[0,1]$. Hence, since by assumption $k<n$, there exists an agent $i \notin S_{k}$. Consider $\tilde{u}_{i} \in U_{i}^{\mathrm{k}}$ such that $\tilde{u}_{i}\left(x, S_{k+1}\right)>0$ for all $x \in[0,1]$. By individual stability $f\left(\tilde{u}_{i}, u_{-i}\right)=\left(z, \bar{S}_{k}\right)$ with $i \in \bar{S}_{k}$. It follows that strategy-proofness is violated at $f(u)$ by agent $i$ who can manipulate via $\tilde{u}_{i}$.

Proof of Theorem 15: The theorem follows from the lemmas below.

Lemma 22 Consider $S \subset N$ and let $f: U_{S}^{k} \times U_{N \backslash S}^{k^{\prime}} \rightarrow[0,1] \times 2^{N}$ be strategy-proof, efficient and outsider independent. (So the domain is one where agents in $S$ always prefer size $k$ and agents in $N \backslash S$ prefer size $k^{\prime}$.) The $f^{G}$ must have a constant size of $k$ or a constant size of $k^{\prime}$. Also, for any $u$ and $u^{\prime}$, if $p\left(u_{i}\right)=p\left(u_{i}^{\prime}\right)$ for all $i$, then $f^{L}(u)=f^{L}\left(u^{\prime}\right)$.

Proof of Lemma 22: The facts that $f^{L}(u)=f^{L}\left(u^{\prime}\right)$ and $f^{G}$ must be of a constant size follow from arguments that are similar to those in the proof of Theorem 12. The fact that the size of $f^{G}$ must be either $k$ or $k^{\prime}$ follows from Pareto efficiency. 
Lemma 23 Let $f: U^{k, k^{\prime}} \rightarrow[0,1] \times 2^{N}$ be strategy-proof, efficient and outsider independent. Then for any $u \in U^{k, k^{\prime}}$ and $u^{\prime} \in U^{k, k^{\prime}}$, if $p\left(u_{i}\right)=p\left(u_{i}^{\prime}\right)$ for all $j$, then $f^{L}(u)=f^{L}\left(u^{\prime}\right)$.

Proof of Lemma 23:The proof is by induction on the agents. First consider agent $i$ who may have preference in $U_{i}^{k, k^{\prime}}$ while all other agents have $u_{j} \in U_{j}^{k}$. Suppose to the contrary that $f^{L}(u)=a \neq b=f^{L}\left(u_{i}^{\prime}, u_{-i}\right)$ for some $u \in U^{k}$ and $u_{i}^{\prime} \in U_{i}^{k^{\prime}}$ with $p\left(u_{i}\right)=p\left(u_{i}^{\prime}\right)$. By Lemma 22, we can assume that $i$ prefers to be in any group at any level and size under both preferences, that either $i$ prefers $a$ at size $k$ or $k^{\prime}$ to $b$ at size $k$ or $k^{\prime}$, or vice versa (in the case where $b=p\left(u_{i}\right)$ ). By strategy-proofness it follows that either $i \in f^{G}(u) \cap f^{G}\left(u_{i}^{\prime}, u_{-i}\right)$, or that $i \notin f^{G}(u) \cup f^{G}\left(u_{i}^{\prime}, u_{-i}\right)$. The second case would violate outsider independence, so it must be the first case. But then this violates strategy- proofness. Iterating on the agents provides the result. I

Lemma 24 Let $f: U^{k} \cup U^{k^{\prime}} \rightarrow[0,1] \times 2^{N}$ be strategy-proof, efficient and outsider independent. Then for any $u \in U^{k} \cup U^{k^{\prime}}$ there exists $i$ such that $f(u)=\left(p\left(u_{i}\right), S\right)$ with $i \in S$ and $|S|$ is of $i$ 's favorite size.

Proof of Lemma 24: Suppose the contrary. Without loss of generality let $f(u)=(a, S)$, where $|S|=k$, while any agent $i \in S$ who has $p\left(u_{i}\right)=a$ (if any) has favorite size $k^{\prime}$. For $j \notin S$ consider $\tilde{u}_{j}$ such that $p\left(\tilde{u}_{j}\right) \neq a$, maintaining the ordering over peaks of the other agents (and varied sizes of groups) as under $u_{j}$ and such that $u_{j}(x, T)<0$ implies $\tilde{u}_{j}(x, T) \leq 0$. It follows from strategy-proofness and outsider independence that $f\left(u_{S}, \tilde{u}_{-S}\right)=(a, S)$. For $j \notin S$ consider $u_{j}^{\prime}$ such that $p\left(u_{j}^{\prime}\right)=p\left(\tilde{u}_{j}\right)$ and $u_{j}^{\prime}(x, T)<0$ for all $x \notin I_{j}\left(p\left(u_{j}^{\prime}\right)\right)$ with $a \notin I_{j}\left(p\left(u_{j}^{\prime}\right)\right)$. It follows from strategy-proofness and outsider independence that $f\left(u_{S}, u_{-S}^{\prime}\right)=(a, S)$. Next for each $i \in S$, consider $u_{i}^{\prime}$ such that $i$ has the same ranking over all choices where $i$ is a consumer as under $u_{i}$, but has $u_{i}^{\prime}(a, S)<0$. It follows from Lemma 22 that $f\left(u^{\prime}\right)=\left(a, S^{\prime}\right)$ where $\left|S^{\prime}\right|=k$. However, this is inefficient since it is Pareto dominated by $(a, \emptyset)$, which is a contradiction.

Lemma 25 Let $f: U^{k} \cup U^{k^{\prime}} \rightarrow[0,1] \times 2^{N}$ be strategy-proof, efficient and outsider independent. Then $f$ is dictatorial.

Proof of Lemma 25: Consider $u \in U^{k} \cup U^{k^{\prime}}$ such that all peaks are distinct. Then by Lemma 24, $f(u)=\left(p\left(u_{i}\right), S\right)$ where $i \in S$, and $S$ is of $i$ 's favorite size, which without loss of generality let be $k$. Change $u_{-i}$ to $u_{-i}^{\prime}$ with the same peaks, and all with favorite size $k^{\prime}$. By Lemma 23, $f^{L}(u)=f^{L}\left(u_{i}, u_{-i}^{\prime}\right)$, and then by Lemma 24 it must be $\left(p\left(u_{i}\right), S^{\prime}\right)$ with $i \in S^{\prime}$, but still $\left|S^{\prime}\right|=k$. We know from Theorem 12 that $f^{L}$ must be determined by a sovereign median rule which always picks a peak of one agent. So consider any $j$ such that there are no peaks of any agent between $u_{j}^{\prime}$ and $u_{i}$. Without loss of generality consider the case where $p\left(u_{j}^{\prime}\right)<p\left(u_{i}\right)$. Let $u_{i}^{\varepsilon} \in U_{i}^{k+}$ be such that $p\left(u_{i}^{e}\right)=\max \left[0, p\left(u_{j}^{\prime}\right)-\varepsilon\right]$ and $u_{i}^{e}(z, T)>u_{i}^{e}\left(y, T^{\prime}\right)$ whenever $i \in T$ and $|T|=k$ and $\left|T^{\prime}\right|=k^{\prime}$ regardless of $z$ and $y$. Since 
this is a generalized median rule, for small enough $\varepsilon$ either $f^{L}(u)=p\left(u_{i}^{e}\right)$ or $=p\left(u_{j}^{\prime}\right)$. By strategy-proofness, it must be that $\left|f^{G}\left(u_{i}^{e}, u_{-i}^{\prime}\right)\right|=k$. Thus, by Lemma 24 it must be that $f^{L}\left(u_{i}^{e}, u_{-i}^{\prime}\right)=p\left(u_{i}^{e}\right)$. It is then clear that $f$ must be dictatorial on the domain where all agents other than $i$ have distinct peaks. Strategy-proofness then easily shows that it must be dictatorial on $U^{k} \cup U^{k^{\prime}}$. 